\title{
Computational and experimental investigation into aerodynamic interference between slender bodies in supersonic flow
}

\author{
R. Chaplin, D. MacManus* \\ Department of Aerospace Sciences, Cranfield University, UK.
}

F. Leopold, B. Martinez, T. Gauthier

French-German Research Institute of Saint-Louis (ISL), France.

T. Birch

Air and Weapon Systems, Defence Science and Technology Laboratory (DSTL), UK.

\author{
* Corresponding Author \\ David MacManus \\ Ph: +44 1234754735 \\ Fax:+44 1234758207 \\ email: d.g.macmanus@cranfield.ac.uk
}

\section{Abstract}

Aerodynamic interference can occur between high-speed bodies when in close proximity. A complex flowfield develops where shock and expansion waves from a generator body impinge upon the adjacent receiver body and modify its aerodynamic characteristics. The aims of this paper are to validate a computational prediction method, to use the predicted solutions to interpret the measured results and to provide a deeper understanding of the associated flow physics.

The interference aerodynamics for two slender bodies were investigated through a parametric wind tunnel study where the effect of axial stagger was investigated for different receiver body incidence angles. Measurements included forces and moments, surface pressures and shadowgraph visualisations. Supporting computational predictions provided a deeper understanding of the underlying aerodynamics and flow mechanisms. Good agreement was found between the measured and predicted interference loads and surface pressures for all configurations.

The interference loads are strongly dependent upon the axial impingement location of the primary shockwave. These induced interference loads change polarity as the impingement location moves aft over the receiver. Distinct interference characteristics are observed when 
the receiver is placed at high positive incidence, where the impinging shock has a strong effect on the crossflow separation location. Overall, the observed interference effects are expected to modify the subsequent body trajectories and may increase the likelihood of a collision.

\section{Keywords}

high-speed aerodynamics, mutual interference, CFD validation, slender body

\section{Nomenclature}

sonic velocity

$\left[\mathrm{ms}^{-1}\right]$

$\mathrm{C}_{\mathrm{p}}$

pressure coefficient

$C_{p}=\frac{p-p_{\infty}}{q_{\infty}}$

$\Delta \mathrm{C}_{\mathrm{p}}$

pressure coefficient difference at given body location

$\Delta C_{p}=C_{p}-C_{p, i s o}$

$\mathrm{C}_{\mathrm{X}, \mathrm{t}}$

measured axial force coefficient

$C_{X, t}=\frac{F_{X}}{q_{\infty} S}$

$C_{x}$

axial force coefficient, corrected to zero base drag

$C_{X}=C_{X, t}+\frac{S_{b}\left(p_{b}-p_{\infty}\right)}{q_{\infty} S}$

$\mathrm{C}_{\mathrm{Z}}$

normal force coefficient

$C_{Z}=\frac{F_{Z}}{q_{\infty} S}$

$\mathrm{C}_{\mathrm{m}} \quad$ pitching moment coefficient (about $\mathrm{x}=0, \mathrm{y}=0, \mathrm{z}=0$ )

$C_{m}=\frac{M_{Y}}{q_{\infty} S D}$

$\Delta \mathrm{C}_{Z} \quad$ normal force interference load at a given $\sigma_{\mathrm{R}}$

$\Delta C_{Z}=C_{Z}-C_{Z, i s o}$

$\Delta \mathrm{C}_{\mathrm{m}} \quad$ pitching moment interference at a given $\sigma_{\mathrm{R}}$

$\Delta C_{m}=C_{m}-C_{m, i s o}$

$\mathrm{dC}_{\mathrm{z}} / \mathrm{dx} \quad$ local normal force coefficient

$\left[\mathrm{m}^{-1}\right]$

D

maximum body diameter at base

[m]

$F_{X} \quad$ receiver body axial force

$[\mathrm{N}]$

$\mathrm{F}_{Z} \quad$ receiver body normal force

$[\mathrm{N}]$

L $\quad$ maximum body length

$[\mathrm{m}]$

$\mathrm{M}_{Y} \quad$ receiver body pitching moment

$[\mathrm{Nm}]$

M Mach number

$M=\frac{U}{a}$

$\mathrm{N}_{\text {fine }} \quad$ total number of cells in fine grid 


\begin{tabular}{|c|c|c|}
\hline $\mathrm{N}_{\text {med }}$ & total number of cells in medium grid & \\
\hline$p$ & static pressure & {$\left[\mathrm{Nm}^{-2}\right]$} \\
\hline $\mathrm{p}_{\mathrm{b}}$ & model base pressure & {$\left[\mathrm{Nm}^{-2}\right]$} \\
\hline$q$ & dynamic pressure & {$\left[\mathrm{Nm}^{-2}\right]$} \\
\hline$\Delta r$ & $\begin{array}{l}\text { distance from generator leading edge to receiver } \\
\text { nearside impingement location }\end{array}$ & {$[\mathrm{m}]$} \\
\hline$r_{\text {eff }}$ & effective grid refinement ratio for an unstructured grid & $r_{\text {eff }}=\left(\frac{N_{\text {fine }}}{N_{\text {med }}}\right)^{1 / 3}$ \\
\hline $\operatorname{Re}_{\mathrm{D}}$ & $\begin{array}{l}\text { freestream Reynolds number based upon base } \\
\text { diameter }\end{array}$ & $\operatorname{Re}_{D}=\frac{\rho_{\infty} U_{\infty} D}{\mu_{\infty}}$ \\
\hline S & receiver body reference area & {$\left[\mathrm{m}^{2}\right]$} \\
\hline$S_{b}$ & receiver body base area & {$\left[\mathrm{m}^{2}\right]$} \\
\hline $\mathrm{T}$ & static temperature & {$[\mathrm{K}]$} \\
\hline U & velocity & {$\left[\mathrm{ms}^{-1}\right]$} \\
\hline$X, Y, Z$ & body fixed axes attached to receiver leading edge & \\
\hline$X_{w}, Y_{w}, Z_{w}$ & $\begin{array}{l}\text { wind axes }\left(X_{w} \text { is aligned in the freestream flow }\right. \\
\text { direction) }\end{array}$ & \\
\hline$\Delta \mathrm{x}$ & axial stagger between bodies & {$[\mathrm{m}]$} \\
\hline$x^{\prime}$ & $\begin{array}{l}\text { axial impingement location of the primary disturbance } \\
\text { measured from receiver leading edge }\end{array}$ & {$[\mathrm{m}]$} \\
\hline $\mathrm{x}_{\text {res }}$ & spatial resolution of PSP & {$[\mathrm{m}]$} \\
\hline$\Delta y$ & spanwise offset between bodies & {$[\mathrm{m}]$} \\
\hline$\Delta \mathrm{z}$ & lateral separation between bodies & {$[\mathrm{m}]$} \\
\hline
\end{tabular}

\section{Greek Symbols}

$\beta \quad$ body sideslip angle

$\delta \quad$ uncertainty for a given parameter

$\mu \quad$ dynamic viscosity

$\theta_{s, G} \quad$ generator bow shock angle, measured from freestream flow axis $\left(X_{w}-Y_{w}\right.$ plane $)$

$\theta_{\text {obl }} \quad$ shock obliqueness angle $\left(\theta_{\mathrm{s}, \mathrm{G}}-\sigma_{\mathrm{R}}\right)$ 


$\begin{array}{lll}\rho & \text { density } & {\left[\mathrm{kgm}^{-3}\right]} \\ \sigma & \text { total incidence angle } & {\left[^{\circ}\right]} \\ \sigma_{\text {eff }} & \text { effective total incidence based on interference load } & {\left[{ }^{\circ}\right]} \\ \Delta & \text { difference of a given parameter from isolated configuration } & \\ \phi & \text { receiver azimuth angle } & \left.{ }^{\circ}\right]\end{array}$

\begin{tabular}{ll}
\multicolumn{2}{l}{ Subscripts } \\
near & receiver nearside conditions \\
iso & isolated conditions \\
0 & stagnation conditions \\
$\infty$ & (inf) freestream conditions \\
R & receiver body \\
G & generator body
\end{tabular}

\section{Introduction}

Aerodynamic interference occurs when two bodies are placed in close proximity in a supersonic flow. This can affect the force and moment characteristics and change the subsequent trajectory of the bodies [1-6]. The interference flowfield is primarily dominated by shock and expansion waves, which originate from an adjacent generator body and impinge upon the primary body of interest (the receiver). The induced changes in static pressure and flow angularity across the impinging disturbances modify both the local and overall aerodynamics of the receiver in comparison to the isolated body case.

There is currently, very little information in the open literature on the effects of mutual interference between slender bodies at high-speed. Previous work reported on this complex research topic can be categorised into three main areas. Many of the papers report the integrated force and moment characteristics of a slender body in close proximity to a second body or flat plate. In most cases there is a very limited explanation about the underlying aerodynamics and about why the integrated effects change with the primary geometric parameters such as body incidence and separation distances. . These investigations were usually experimental and focussed on receiver bodies which remained at low incidence. The second category of previous work studied the more detailed aspects of shock-body interactions. These were primarily wind tunnel investigations which used simplified experimental arrangements such as planar shockwaves impinging onto axisymmetric bodies. Finally, there are a small number of computational studies which attempt to understand the problem of high-speed mutual interference. The important contributions from these three areas are now briefly summarised and used to place the current work in context. 
An early experimental investigation by Gapcynski and Carlson [3] examined two axially aligned bodies of revolution at a freestream Mach number of $M_{\infty}=2.01$. These researchers reported changes in normal force coefficient of up to $\Delta \mathrm{C}_{\mathrm{z}}=0.1$ as a result of the interference flowfield between the bodies. Another study showed that a planar shock impinging on a cone-cylinder body at zero incidence induced changes in normal force and pitching moment coefficient in the range of $-0.22<\Delta \mathrm{Cz}<0.048$ and $-0.39<\Delta \mathrm{Cm}<-0.20$ [1]. The pitching moment values reported by Wilcox [1] have been transformed to be consistent with the definition used throughout this paper. These induced changes were found to increase when the receiver body was placed at an incidence of $\sigma=15^{\circ}$. Interference effects of this order are likely to modify the trajectory of the slender body [6]. This would become significant if the slender body pitches,or translates, towards the generator since this may subsequently lead to a collision. A more recent study, which investigated two axially aligned slender bodies at zero incidence, found that the polarity of the resulting pitching moment load was strongly dependent upon the lateral separation between the bodies [7]. It has been noted by several authors that the mutual interference flowfield and resulting induced pitching moment could potentially have a notable effect on the trajectory of both bodies $[3,4,8,9,10]$.

In addition to studies which report the overall interference loads, it is important to understand the detailed underlying flow physics. Of particular interest are the shock-body interactions which have been studied previously for a number of configurations [7, 8, 11-15]. Brosh et al. [14] and Hung [2] investigated a wedge-generated shock impinging on a cylinder at $M_{\infty}=3$ which showed that the shock footprint, in terms of local pressure rise, decreased as the shock diffracted around the body. The difference between the strength of the nearside and farside regions of augmented pressure significantly affect the local normal force distribution over the body. Finally, the nearside pressure rise also resulted in a local boundary-layer separation and a double-reflected shock structure around the primary induced separation bubble. Both studies found that due to the induced circumferential pressure gradient a strong crossflow occurred which resulted in a local separation on the farside of the receiver body.

Finally, in addition to the mainly experimental studies discussed above, there have been some computational studies of mutual interference for slender bodies at high speeds $[14,16,17]$. Brosh et al. [14] and Hung [16] conducted one of the few CFD validation exercises using a thin-layer approximation of the 3D RANS equations to simulate the experimental set-up tested by Brosh et al. [14]. This configuration featured a planar shock impinging onto a cylinder which was at zero incidence. Hung focussed on assessing the ability of the CFD solver to predict the complex shockwave boundary-layer interactions around the cylinder. It was reported that the prediction of surface axial pressure distributions on the cylinder were in good agreement with the measurements. However, some of the viscous flow structures were not predicted well by the CFD. In particular, the flow topology on both the nearside and farside of the cylinder which included induced flow separations were not predicted accurately. More recently, Volkov and Derunov [7] investigated the effect of lateral separation between two axially aligned slender bodies and found reasonably good agreement between the measured and predicted loads. Finally, both Malmuth and Shalaev [8] and Fedarov et al. [13] have reported theoretical approaches to the prediction of overall loads on two slender bodies in close proximity.

It is clear from the discussion above that there are a number of knowledge gaps related to the understanding and prediction of aerodynamic interference between slender bodies at 
supersonic speeds. Specifically, there has been little or no attention to date on the impact of receiver body incidence or on the strength of the disturbance flowfield. Moreover, although the effect of the axial impingement location of the primary shock has been discussed by some researchers, this has often been achieved by changing the lateral separation between the bodies where there is a corresponding change in the strength of the impinging shock. The current investigation maintains a constant lateral separation throughout, in order to decouple the effect of axial shock impingement location and disturbance field strength. Also, the computational studies to date have not been comprehensive enough to understand the underlying flow physics of the problem, especially for bodies at incidence.

There are two main aims of this paper which will address some of the poorly understood aspects of high-speed mutual interference. The first is to quantify and understand the interference aerodynamics associated with slender bodies in various multi-body arrangements. This includes the effect of receiver body incidence, axial stagger and disturbance field strength which have not been adequately investigated previously. The second aim is to assess the capability of modern CFD methods to predict multiple slender body, high-speed flowfields. This involves the comparison of the measured and predicted flowfield structures and interference loads as well as surface pressure distributions. This paper will validate the CFD method for configurations which have not been reported previously, for example where the bodies are at high incidence.

Given the complexity of the interference flowfield, the strategy of the current research is to use a mutually enhancing and comprehensive combination of both experiments and computational results to develop a deeper understanding of the underlying flow physics of the problem. The first step in meeting these aims was to conduct a wind tunnel study where the primary measurements were the receiver forces and moments, surface pressures and shadowgraph visualisations. The experiments investigated the effect of the receiver body incidence, the disturbance field strength and the axial stagger between the bodies. A complimentary step was to compute a comprehensive matrix of computational predictions to both help design the experiments as well as to interpret the measurements.

\section{Experimental method}

\subsection{Model description and wind tunnel arrangement}

The wind tunnel tests were performed using two bodies placed in close proximity. The body of interest is designated as the receiver which is used to obtain the quantitative measurements. The receiver comprises a 3.5D tangent ogive forebody and a cylindrical afterbody. It has an overall length of $L / D=7.358$, where $D$ is the base diameter of $D=20 \mathrm{~mm}$ (Fig. 1). The other body is considered as the generator which is used to create the disturbance flowfield. Two axisymmetric generator bodies were used and no measurements were taken on the generator bodies. The first is a sharp nosed generator which is identical to the receiver body (Fig. 1). The second generator is a blunt-nosed body with the same overall length as the sharp generator $(\mathrm{L} / \mathrm{D}=7.358, \mathrm{D}=20 \mathrm{~mm})$ but includes a hemi-spherical forebody. 

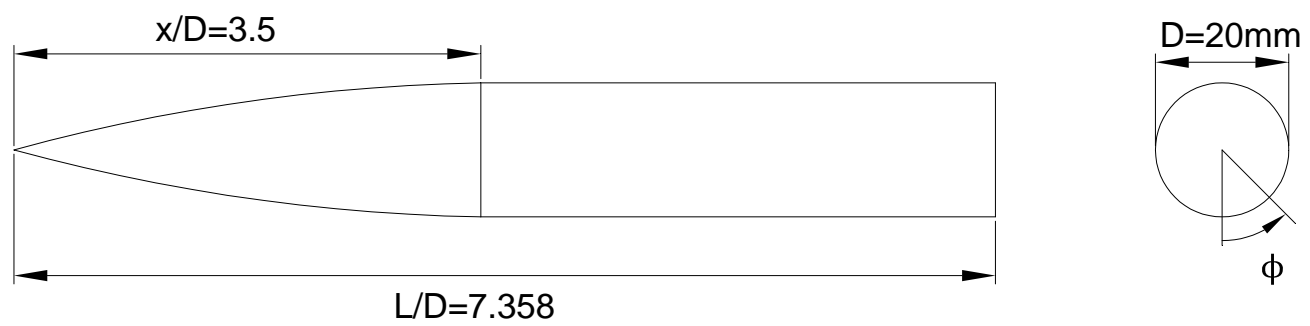

Fig. 1 Schematic of the receiver body and definition of the azimuth angle $(\phi)$

The experiments were performed in the $0.2 \mathrm{~m} \times 0.2 \mathrm{~m}$, S20 supersonic wind tunnel at the French-German Research Institute of Saint-Louis (ISL). This is a blowdown tunnel with a typical run time of $50 \mathrm{~s}$. The working section freestream flow conditions were a Mach number of $\mathrm{M}_{\infty}=2.43$, a total pressure of $\mathrm{p}_{0}=0.675 \mathrm{MPa}$ and a total temperature of $\mathrm{T}_{0}=293 \mathrm{~K}$. This provided a freestream Reynolds number based on body diameter of $R e_{D}=1.4 \times 10^{6}$. No boundary-layer transition devices were used for the interference tests presented in this paper. However, additional measurements for the isolated receiver body were performed over an incidence range of $-15 \leq \sigma \leq 15^{\circ}$ using a wire transition strip attached $2 \mathrm{~mm}$ from the leading edge. These tests demonstrated a negligible effect on the normal force and pitching moment coefficients in comparison to the natural transition tests [6]. Moreover, there was typically only a small increase in axial force of $\Delta \mathrm{C}_{\mathrm{X}, \mathrm{t}}=0.01$ which is approximately $3 \%$ of the total measured which remained approximately constant for all incidence values $\left(C_{X, t}=0.3\right)$. No specific boundary-layer transition tests were conducted to provide evidence of the state of the boundary-layer for the natural and transition-fixed wind tunnel runs. However, since the normal force and pitching moment measurements characteristics of the body are sensitive to the leading separation location over this incidence range and since these were indistinguishable between the natural and transition-fixed tests, it is therefore assumed that the boundary-layer was naturally turbulent during these experiments.

Multi-body testing was performed using a dual support system (Fig. 2). A traverse mechanism allowed both the upper generator body and the lower receiver body to be translated relative to one another in the streamwise direction. The receiver body incidence was controlled using the lower support sting, which rotated about a centre of rotation point $1.65 \mathrm{D}$ upstream of the base on the body centreline. Two $D_{s}=16 \mathrm{~mm}$ diameter stings connected each body to their respective supports (Fig. 2).

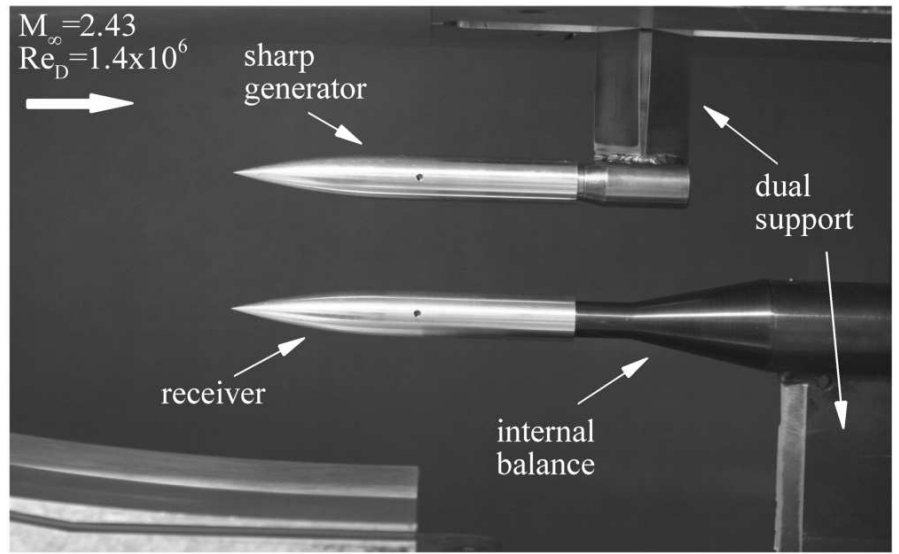

Fig. 2 Receiver body set-up adjacent to the sharp generator

Each multi-body configuration is defined by the relative lateral separation $(\Delta z)$, spanwise offset $(\Delta y)$ and axial stagger $(\Delta x)$ (Fig. 3). The spanwise offset between the bodies and the 
body sideslip angles were both zero in all cases $\left(\Delta y=0, \beta=0^{\circ}\right)$. The lateral separation $(\Delta z)$ is defined as the distance between the generator centreline and the centre of rotation for the receiver body. Moreover, the lateral separation $(\Delta z / D=2.94)$ and generator incidence $\left(\sigma_{G}=0^{\circ}\right)$ also remained fixed throughout.

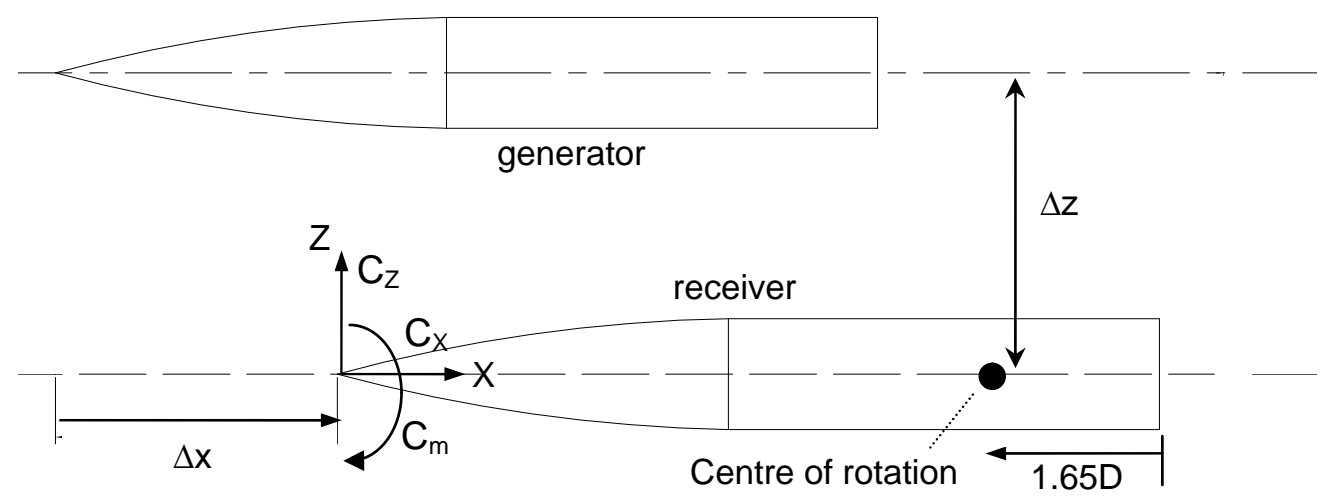

Fig. 3 Definitions of positive axial stagger $(\Delta x)$ and positive lateral separation $(\Delta z), \Delta y$ into page.

\subsection{Experiment procedure and measurements}

For the multi-body configurations, the receiver body incidence was typically varied between $15 \leq \sigma_{R} \leq 15^{\circ}$. A 5 s settling period was employed at the start of each tunnel run. At each incidence, the receiver body paused and the forces and moments were measured using an ABLE MKXIV 6-component, internal balance. The force and moment balance output was filtered using a $5 \mathrm{~Hz}$ low-pass filter and the data was acquired at a sample rate of $100 \mathrm{kHz}$ with a sample duration of $2 \mathrm{~s}$. A shadowgraph image was also taken at each incidence setting. A light source was focused using a $0.38 \mathrm{~m}$ diameter lens and the illuminated flowfield was projected onto a transparent screen on the opposite tunnel window where a still image was taken with a PCO Sensicam qe camera. Finally, Pressure Sensitive Paint (PSP) measurements on the receiver were taken for a subset of configurations. A full description of the PSP set-up and measurement procedure is presented in Chaplin [6].

This same experiment procedure was used for different axial stagger configurations, different generator bodies as well as the isolated receiver configurations. The overall test matrix comprised configurations with an axial stagger of $\Delta x / D=-1.65,0,1.67,2.68,3.679$, a receiver incidence range of $-15 \leq \sigma_{R} \leq 15^{\circ}$ as well as the use of a sharp and blunt generator.

\subsection{Experimental uncertainly}

The experimental uncertainties were calculated using the approach of Taylor [18] and are mostly expressed as a fractional uncertainty of the measured value (Table 1). All measurement uncertainties were estimated from systematic and random sources of error which included the instrument calibration, instrument accuracy given by the manufacturer and the data acquisition resolution. The uncertainty in the PSP measurement is based on a typical difference between the measured PSP and an in-situ static pressure measurement taken on the balance near the model base.

\begin{tabular}{llll}
\hline Tunnel arrangement & Freestream conditions & Forces and moments & PSP
\end{tabular}




\begin{tabular}{llllllll}
\hline$\Delta \mathrm{x} / \mathrm{D}$ & $\pm 0.7-5.7 \%$ & $\mathrm{M}_{\infty}$ & $2.43 \pm 1.2 \%$ & $\mathrm{C}_{\mathrm{Z}}$ & $\pm 0.6 \%$ & $\mathrm{p}$ & $\pm 10 \%$ \\
$\Delta \mathrm{z} / \mathrm{D}$ & $\pm 0.1 \%$ & $\mathrm{Re}_{\mathrm{D}}$ & $1.4 \times 10^{6} \pm 0.4 \%$ & $\mathrm{C}_{\mathrm{m}}$ & $\pm 0.6 \%$ & & \\
$\sigma_{\mathrm{R}}$ & $\pm 0.1^{\circ}$ & & & $\Delta \mathrm{C}_{\mathrm{Z}}$ & $\pm 0.9 \%$ & & \\
$\mathrm{x}^{\prime} / \mathrm{L}$ & \pm 0.01 & & & $\Delta \mathrm{C}_{\mathrm{m}}$ & $\pm 0.9 \%$ & & \\
\hline
\end{tabular}

Table 1 Summary of the measurement uncertainties

\section{Computational method}

\subsection{Solver description}

All computational predictions were conducted using the flow solver Cobalt [19]. This is an unstructured, implicit solver based on a finite volume formulation and further details are reported by Strang et al. [20]. Steady-state viscous predictions were performed which solved the Reynolds averaged Navier-Stokes (RANS) equations and modelled turbulence using Menter's 2-equation SST turbulence model [21]. The solutions were obtained using $2^{\text {nd }}$ order spatial accuracy and the wind tunnel freestream conditions were specified as initial conditions.

\subsection{Computational grids}

All the 3D computational grids were created using the grid generation software Gridgen [22] and were based on a hybrid meshing approach. The computational domain modelled onehalf of the flowfield since the body sideslip angles were both zero. This helped to minimise computational requirements. The surfaces of the receiver and generator bodies were generated using a CAD database of the wind tunnel models (with the model attachment hole removed). The computational domain extended from a short distance upstream of the leading edge of the foremost body to approximately 3D downstream of the base of the aftmost body. The base and supporting sting were also included for each body where appropriate. The surrounding flowfield domain was arranged so that the bow shock of both bodies exited through the downstream outlet face. Typical grid sizes were in the order of 15$19 \mathrm{~m}$ cells for the half-flowfield domain.

The hybrid grids contained both structured and unstructured blocks and used hexahedral, tetrahedral and prismatic cell types. The body surfaces, and a region close to each body, were gridded using structured cells in order to achieve an appropriate level of boundarylayer resolution. A first cell spacing in the radial direction adjacent to each solid wall was chosen to ensure an average $y^{+}$value over the body of less than or equal to one [19]. A progression ratio of approximately 1.1 was used to cluster the grid points radially outward from the body surface and typically, 25-30 cells spanned the boundary-layer maximum thickness [23]. In this structured portion of the grid, a typical number of cells was 300 (longitudinal) $\times 140$ (radial) $\times 260$ (circumferential). Special care was taken to ensure there was smooth cell size progression across all block boundaries.

\subsection{Boundary conditions}

All body and sting surfaces were defined as no-slip solid walls (Fig. 4). A symmetry condition was applied on the wind axes centreline $\left(X_{w}-Z_{w}\right)$ since the sideslip of the bodies were zero. Both the inlet and farfield boundary conditions were prescribed as fixed 
supersonic inflows at the wind tunnel freestream operating conditions. The outlet boundary values were calculated using a modified Riemann invariants condition [19].

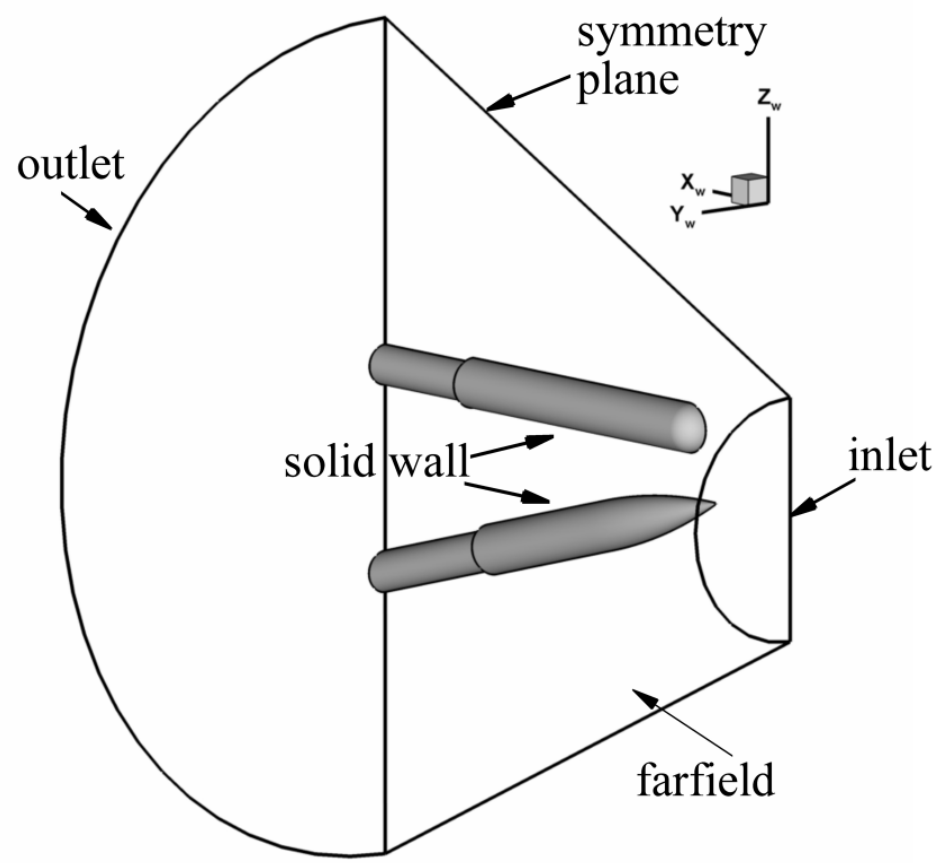

Fig. 4 Illustration of boundary conditions applied in the computational predictions

\subsection{Computational uncertainty}

A discussion of the iterative convergence and spatial discretisation errors in the computational results presented in this paper are given below. All other sources of computational uncertainty such as geometry modelling errors, computer round-off and programming errors are assumed to be negligible.

\subsubsection{Iterative convergence}

Iterative convergence was assessed by examination of the solution residuals and the forces and moments on the receiver body over the solution time. For all computational solutions, satisfactory iterative convergence of the solution residuals was observed and the forces and moments converged to within $0.5 \%$ of the reported values.

\subsubsection{Grid convergence}

A selection of configurations which are representative of the dataset as a whole were further investigated to assess the solution sensitivity to spatial resolution and to provide an estimate of the ordered discretisation error using the approach of Roache [24]. A grid convergence study was completed for each of the selected configurations over three grid levels. For the hybrid grids used in this investigation it was necessary to use an effective grid refinement ratio [24]. This was based on the relative total grid sizes and was approximately $r_{\text {effi }} \approx 1.5$ for this investigation (Eqn (1) shows $r_{\text {eff }}$ between the fine and medium grid levels).

$$
r_{\text {eff }}=\left(\frac{N_{\text {fine }}}{N_{\text {med }}}\right)^{1 / 3}
$$

The integrated force and moment coefficients $\left(\mathrm{C}_{\mathrm{x}}, \mathrm{C}_{2}, \mathrm{C}_{\mathrm{m}}\right)$ were used as the comparison parameters. A typical grid convergence index between the fine and medium grids was less 
than $0.5 \%$. This is considered a reasonable estimate of the discretisation error and the results of the fine grid solutions are presented this paper. Overall, the fine grid solutions were considered to be grid independent.

\section{Results}

The results in this paper are presented in two stages. The objective of the first stage is to assess the capability of the computational method. The objective of the second stage is to use the computational results in conjunction with the measurements to develop an understanding of the underlying flow characteristics and mechanisms.

The overall capability of the computational prediction method is assessed for a range of configurations which include different receiver incidence angles and disturbance field strengths (§4.1). The configurations chosen for analysis in this first section are representative of the range of configurations and flow conditions across the test matrix. These validation comparisons primarily focus on the force and moment data as well as the flow structure and quantitative surface pressure distributions.

Following the validation of the computational method, the complementary experimental and computational datasets are used to analyse the effect of axial stager on the interference aerodynamics where there are notable changes in the underlying flowfields (§4.2-4.4). Finally, a particular configuration which features a complex viscous interaction is also investigated in detail in §4.5. In these latter sections, the computational data is vital to understand the underlying flow physics and to interpret the measured results as this provides additional flowfield information.

\subsection{Validation of the computational method}

The wind tunnel study provides an extensive dataset which is used to validate the computational prediction method. This dataset includes force and moment measurements, shadowgraph visualisations as well as surface pressure measurements for selected configurations. This section assesses the ability of the computational method to predict the measured results for a number of cases which are representative of the complete dataset. Additional information on the aerodynamics of the configurations reported in this section are presented in Chaplin [6].

\subsubsection{Flowfield structure}

The first validation objective is to assess the capability of the CFD to predict the relevant flow features in the interference flowfield. Two relatively simple configurations are chosen for an initial analysis where the receiver body is placed at an incidence angle of $\sigma_{R}=8^{\circ}$. In the first configuration, the sharp generator is placed at an axial stagger of $\Delta x / D=1.67$ upstream of the receiver (Fig. $5 \mathrm{a}$ ). In the second, the blunt generator is placed at an axial stagger of $\Delta x / D=-$ 0.53 downstream of the receiver (Fig. 5 b). In both configurations, the prominent flow features include the generator bow shock which impinges on the receiver nearside $\left(\phi=180^{\circ}\right)$. The axial impingement location of the primary shock is almost the same for both configurations with only a small change from $x^{\prime} / L=0.25$ for the sharp generator configuration (Fig. $5 \mathrm{a}$ ) to $x^{\prime} / L=0.28$ for the blunt generator configuration (Fig. $5 \mathrm{~b}$ ). A portion of the impinging shock reflects back towards the generator body and the remainder diffracts around the body to the receiver farside $\left(\phi=0^{\circ}\right)$, Fig. 5 . Moreover, in both cases the expansion waves which emanate from the generator forebody also impinge on the receiver 
nearside surface, downstream of the primary impingement location. Visualisations constructed from predicted contours of $|\nabla \rho|$ on the symmetry plane are shown for these two configurations in Fig. 6. In both cases, the CFD captures all of the important disturbance waves between the bodies (Fig. 6 a) and predicts the axial impingement location to within $1 \%$ of the measured values (Fig. 6 b).

(a)

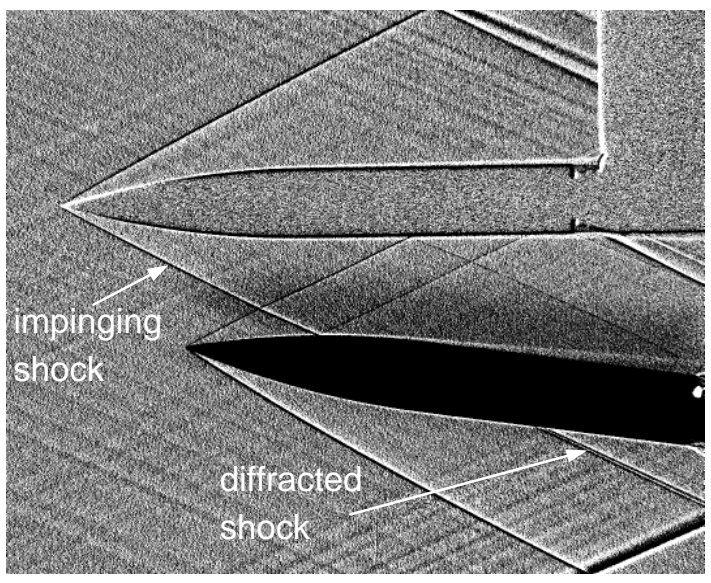

(b)

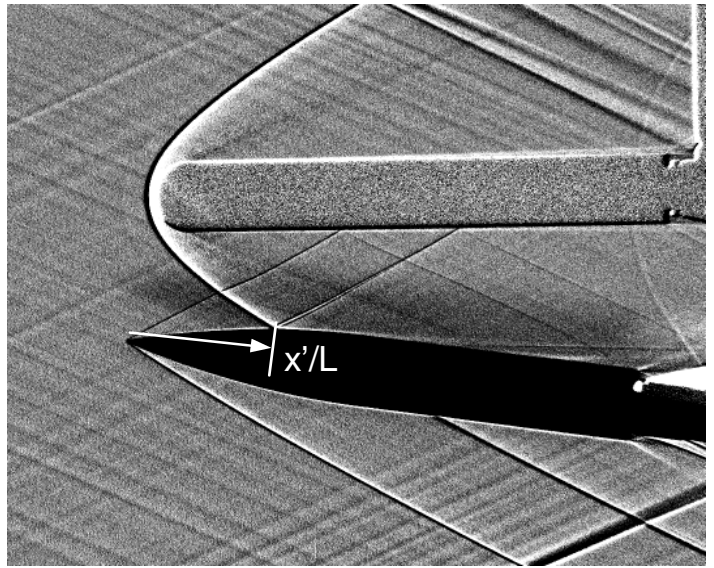

Fig. 5 Measured shadowgraph visualisation: $\Delta z / D=2.94 \sigma_{G}=0^{\circ}, \sigma_{R}=8^{\circ}$ (a) sharp generator $\Delta x / D=1.67$, (b) blunt generator $\Delta x / D=-0.53$, definition of axial impingement location $\left(x^{\prime} / L\right)$

(a)

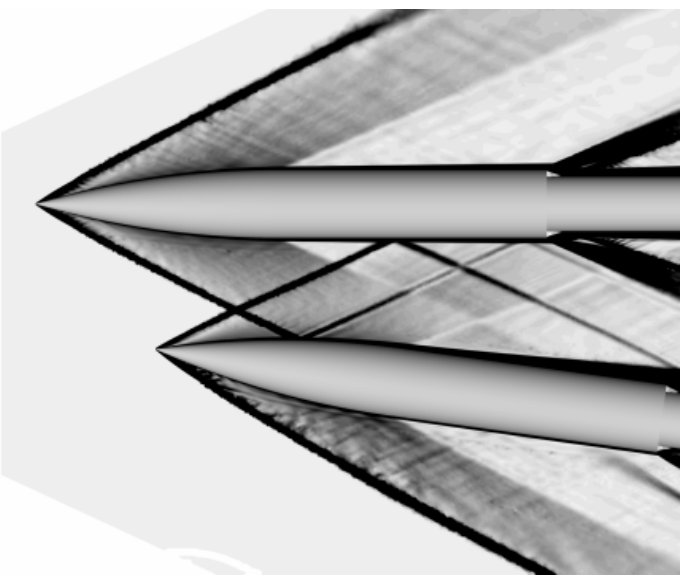


(b)

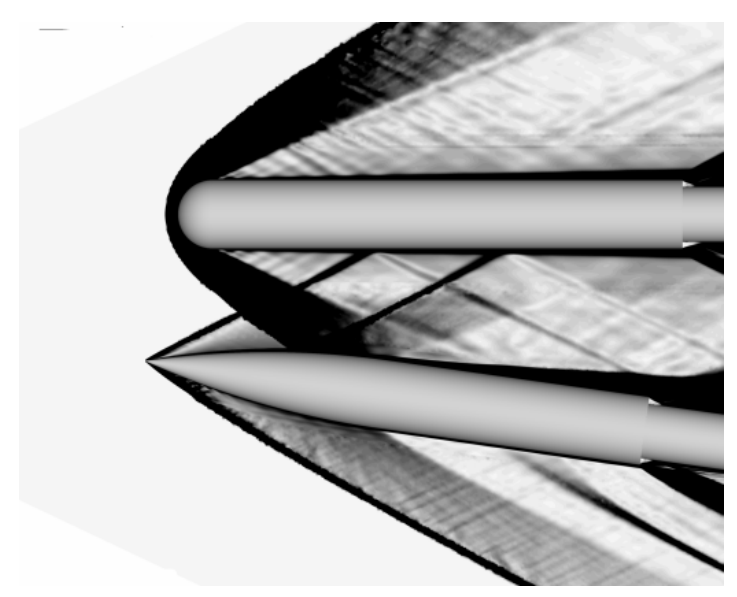

Fig. 6 Predicted contours of $|\nabla \rho|$ on symmetry plane: $\Delta z / D=2.94 \sigma_{G}=0^{\circ}, \sigma_{R}=8^{\circ}$ (a) sharp generator $\Delta \mathrm{x} / \mathrm{D}=1.67$ and $(\mathrm{b})$ blunt generator $\Delta \mathrm{x} / \mathrm{D}=-0.53$

\subsubsection{Interference loads}

The interference loads $\left(\Delta \mathrm{C}_{\mathrm{z}}, \Delta \mathrm{C}_{\mathrm{m}}\right)$ are defined as the measured difference between the receiver loads in a multi-body configuration and those for the isolated configuration as a result of the disturbance flowfield (e.g. $\Delta \mathrm{C}_{z}=\mathrm{C}_{z}-\mathrm{C}_{Z \text {,iso }}$ for normal force). In this section, a validation assessment is completed which compares the measured and predicted interference loads for two cases. The first is where the receiver is adjacent to the sharp generator at an axial stagger of $\Delta x / D=1.67$ (Fig. 7 a) and the second for an 'equivalent' blunt generator case at an axial stagger of $\Delta x / D=-0.53$ (Fig. $7 \mathrm{~b}$ ). These cases are selected as they encompass the range of aerodynamic features experienced during the current investigation. They also enable a comparison of the effect of receiver incidence and disturbance field strength.

The 'equivalent' blunt generator configuration is selected so that the primary shock impingement location on the receiver body at zero incidence $\left(\sigma_{R}=0^{\circ}\right)$ is the same as that for the sharp generator configuration. The difference in the shape of leading edge shock structures between the two generator bodies means that a different axial stagger arrangement is required to meet this condition. In all cases, the nominal axial stagger arrangements remain unchanged when the receiver is pitched through the incidence range under investigation. The axial stagger is not individually set for each incidence angle, instead it is nominally set for the two bodies at zero incidence and then the receiver incidence is subsequently varied.

The comparison between the sharp and blunt generator bodies provides information about the sensitivity of the interference loads to the strength of the disturbance flowfield. There are two main differences in the disturbance flowfield induced by the sharp and blunt generator bodies. The first is the stronger bow shockwave induced by the hemi-spherical forebody of the blunt generator (see section 2.1 for details about the geometric differences between the two generator bodies). It should be noted that the generator bow shock angle $\left(\theta_{\mathrm{s}, \mathrm{G}}\right)$, measured from the freestream flow axis $\left(X_{w}-Y_{w}\right.$ plane $)$ is similar for the sharp $\left(\theta_{s, G}=28.2^{\circ}\right)$ and blunt generators $\left(\theta_{\mathrm{s}, \mathrm{G}}=28.6^{\circ}\right)$ if this measurement is taken more than $\Delta \mathrm{z} / \mathrm{D}=1.5$ from the generator centreline. Secondly, the strength and extent of the expansion wave fan induced by the generator forebody is significantly different between the sharp and blunt cases. In the blunt generator case, the expansion wave fan is stronger but its spatial extent is less than the sharp generator case. 
In general, the magnitude of the interference effects are greatest when the body is placed at high positive or negative incidence in comparison to the zero incidence configuration (Fig. 7 a). When a stronger disturbance field strength is induced by the blunt generator, there is only a modest amplification in the interference loads and the trend with respect to receiver incidence is largely unchanged (Fig. 7 b). This is in spite of the three-fold increase in the primary impinging shock strength when the blunt generator is used in comparison to the sharp generator body. The main differences between the sharp and blunt generator cases are observed at large negative incidence. For the configurations with the receiver at zero or high positive incidence there is a balance between the different local interaction and the overall interference loads are primarily insensitive to disturbance field strength.

The predicted effect of receiver incidence on the interference loads is compared to the measurements for a subset of incidence angles $\left(\sigma_{R}=0^{\circ}, \pm 8^{\circ}, \pm 15^{\circ}\right)$ which span the measured range $\left(\sigma_{R}= \pm 15^{\circ}\right)$. For both the sharp and blunt generator cases, good agreement is observed between the measured and predicted interference loads across a wide range of incidence angles and thus different underlying flow physics (Fig. 7 a,b). A typical maximum difference between the measurements and predictions is in the order of $\Delta C_{X}=0.01, \Delta C_{Z}=0.02$ and $\Delta \mathrm{C}_{\mathrm{m}}=0.1$.

(a)

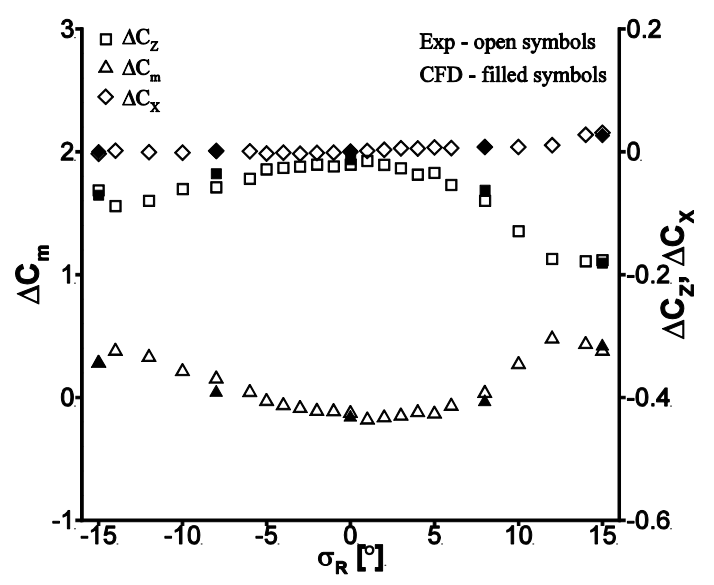

(b)

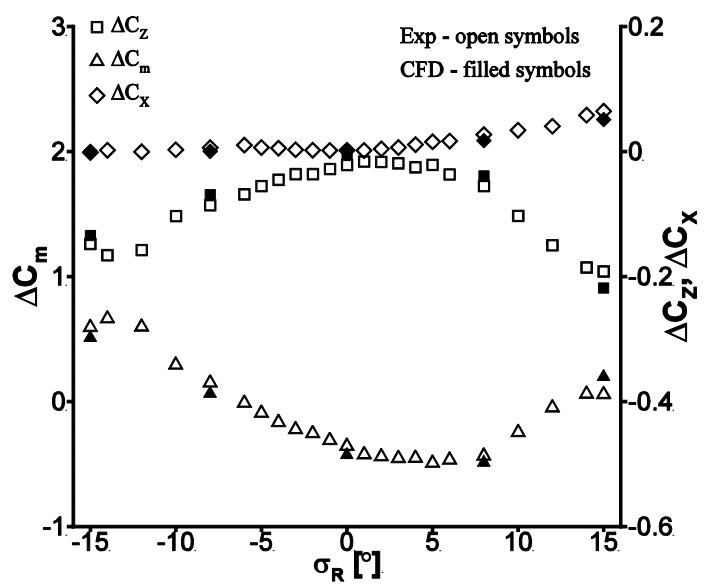

Fig. 7 Comparison between measured and predicted interference loads: $\Delta z / D=2.94, \sigma_{G}=0^{\circ}$, (a) sharp generator $\Delta x / D=1.67$, (b) blunt generator $\Delta x / D=-0.53$. Error bars omitted for clarity 


\subsubsection{Surface pressure distributions}

An additional validation objective is to assess whether the observed agreement between the predicted and the measured interference loads is based on resolution of the correct flow physics. This is important because the underlying flow physics for even an isolated slender body can change appreciably across the range of incidence angles presented in Fig. 7. For example, as the receiver incidence increases from $\sigma_{R}=0^{\circ}$ to $\sigma_{R}=15^{\circ}$, the effects of streamwise load distribution, crossflow boundary-layer separation and vortex development become more notable [6]. These complex features are modified by the impinging shock and expansion waves, which in turn lead to a change in the local aerodynamics and ultimately the overall interference loads. Flowfield comparisons (Fig. 5 and Fig. 6) demonstrate that the CFD successfully predicts the location of the impinging waves and the diffracted shock path around the receiver body. The receiver surface static pressure field is dominated by these impinging waves which, in comparison with the isolated body configuration, induce regions of augmented or diminished pressure $\left(\Delta C_{p}=C_{p}-C_{p, i s o}\right)$. The PSP measurement technique is therefore a useful tool since it offers the benefit of quantitative pressure measurements but more importantly it provides global surface coverage of the receiver body with very good spatial resolution $\left(x_{\text {res }} / D=0.0065\right)$. This is a key aspect of this investigation as it will help to identify and quantify the regions of differential pressure which are the main contributor to the overall interference loads [8].

The measured and predicted pressure distributions on the unwrapped receiver surface are compared in Fig. 8 for a configuration where the receiver is at $\sigma_{R}=0^{\circ}$. This configuration includes the sharp generator with an axial stagger of $\Delta x / D=1.67$ (as reported in Fig. 7 a). There is good qualitative agreement between the two set of results. The CFD predicts the location and magnitude of the nearside pressure rise (Fig. 8 a,b). Furthermore, there is also good quantitative agreement between the measured and predicted nearside pressure distribution (Fig. $8 \mathrm{c}$ ), where the impact of the impinging shockwave and expansion waves can been seen clearly. The CFD also predicts the diffracted shock path around the body as highlighted by the local regions of augmented pressure (Fig. 8 b). An important aspect of the flowfield is observed in the area towards the rear of the body where there is a rise in the pressure on the receiver farside $(x / L>0.8$, Fig. 8 a). This is associated with the crossing of the diffracted shocks across the body symmetry plane and has a notable effect on the overall interference force and moment loads. Although the imposed disturbance field strongly affects the local aerodynamics, the changes in the overall loads are small. This is because, in this particular configuration, the induced local pressure changes on the nearside and farside of the receiver body balance against each other. For this configuration at $\sigma_{R}=0^{\circ}$ the measured interference loads are $\Delta C_{z}=-0.02$ and $\Delta C_{m}=-0.13$ and the predicted interference loads are $\Delta C_{Z}=-0.013$ and $\Delta C_{m}=-0.16$ respectively (Fig. 7 ).

For the configuration with the receiver at $\sigma_{R}=0^{\circ}$ the effect of disturbance field strength is investigated by comparing the surface pressure distributions which arise with the sharp and blunt generators. As described in §4.1.2, the axial stagger has been adjusted so that the primary shock impingement point is conserved between the two cases. In general, the measured flow structures are similar for both cases (Fig. 8a, Fig. 9a). Moreover, good agreement is also observed between the measured (Fig. 9 a) and predicted (Fig. 9 b) pressure distributions for the blunt generator configuration. As expected, the stronger impinging shock increases the magnitude of the nearside pressure rise in comparison to the configuration involving the sharp generator. However, the farside pressure rise is also 
greater for the blunt generator configuration. As a result, the overall interference loads are similar to those found when the sharp generator is used (Fig. 7 b). The overall interference loads for this configuration are therefore primarily insensitive to disturbance field strength because the amplified local interactions balance one another.

(a)

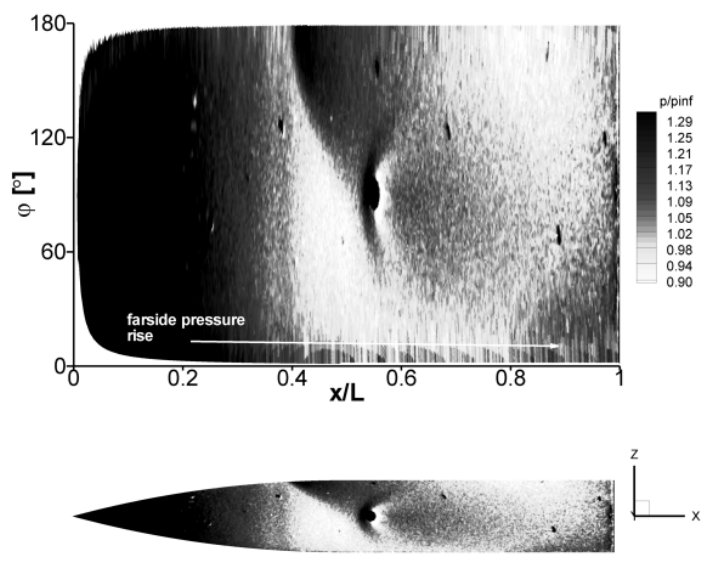

(b)

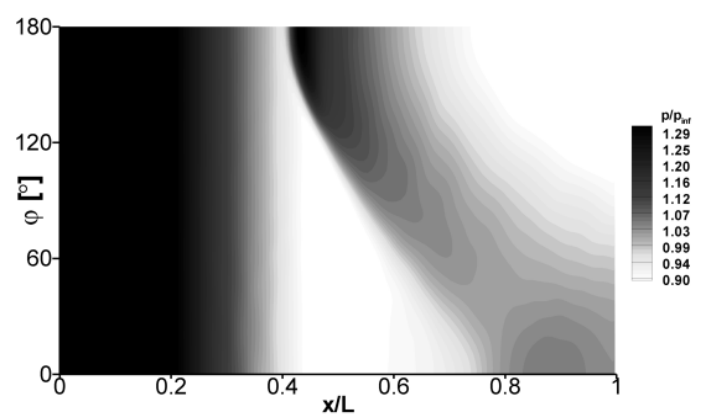

(c)

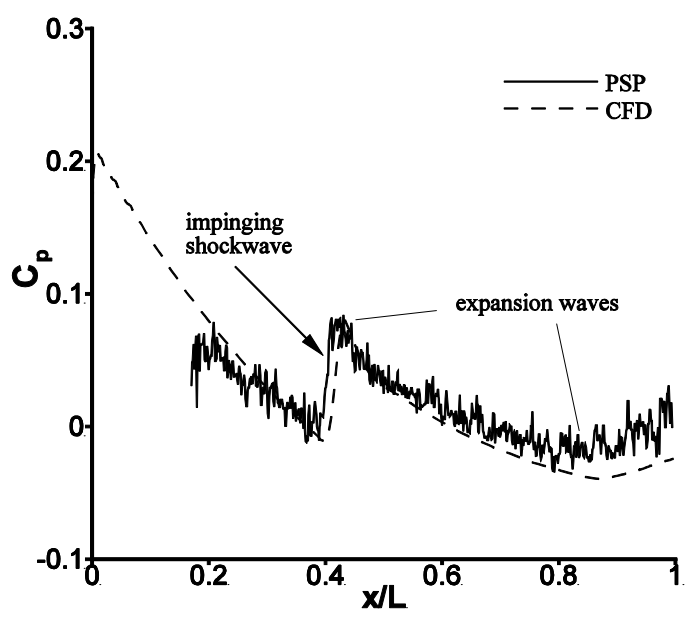


(d)

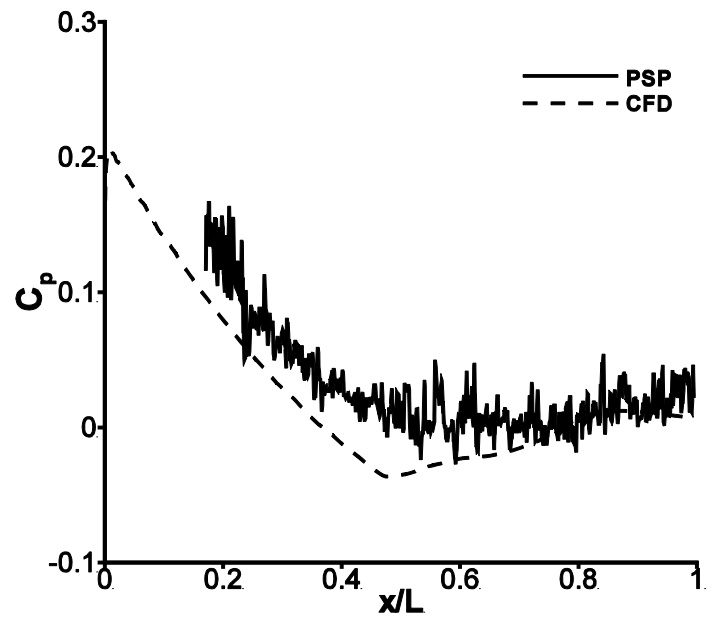

Fig. 8 (a) Measured and (b) predicted surface pressure distributions, quantitative PSP and CFD comparison of (c) nearside pressure distribution $\left(\phi=177^{\circ}\right)$, (d) farside pressure distribution $\left(\phi=3^{\circ}\right)$ : sharp generator, $\Delta \mathrm{x} / \mathrm{D}=1.67 \Delta \mathrm{z} / \mathrm{D}=2.94, \sigma_{\mathrm{R}}=0^{\circ} \sigma_{\mathrm{G}}=0^{\circ}$

(a)

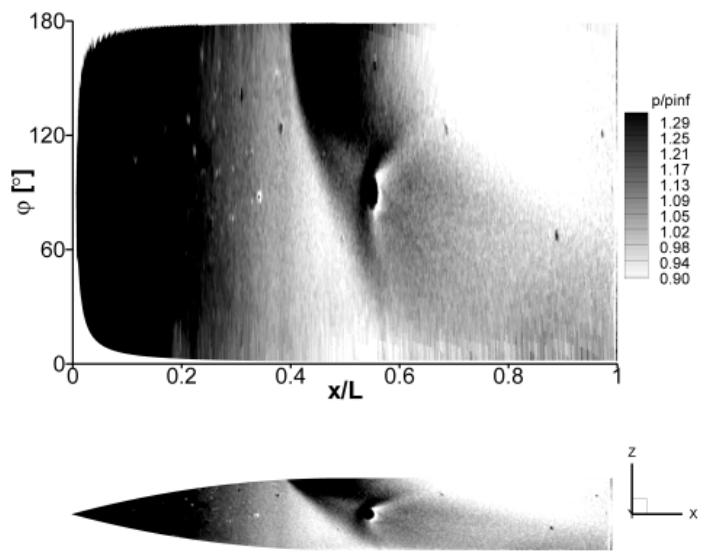

(b)

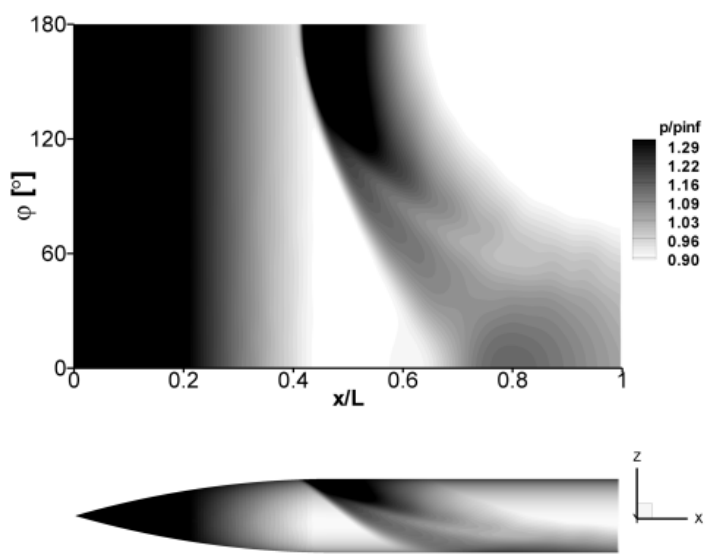


(c)

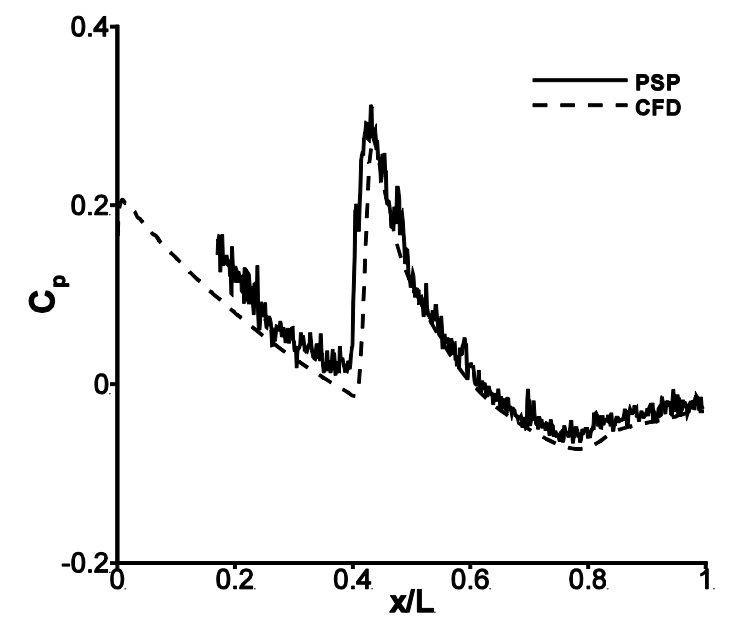

(d)

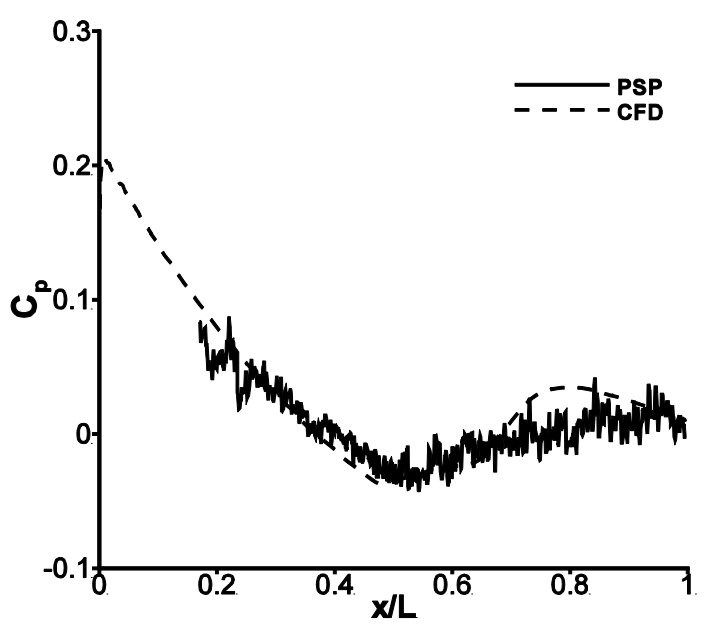

Fig. 9 (a) Measured and (b) predicted surface pressure distributions, quantitative PSP and CFD comparison of (c) nearside pressure distribution $\left(\phi=177^{\circ}\right)$, (d) farside pressure distribution $\left(\phi=3^{\circ}\right):$ : blunt generator $\Delta \mathrm{x} / \mathrm{D}=-0.53 \Delta \mathrm{z} / \mathrm{D}=2.94, \sigma_{\mathrm{R}}=0^{\circ} \sigma_{\mathrm{G}}=0^{\circ}$

When the receiver is placed at high positive incidence $\left(\sigma_{R}=15^{\circ}\right)$ and alongside the sharp generator relatively large interference loads are measured, $\Delta C_{Z}=-0.18$ and $\Delta C_{m}=0.38$ (Fig. 7 b). The measured surface pressure distribution (Fig. 10) shows a characteristically different flowfield to the previous configurations studied at zero incidence (Fig. 8a and Fig. 9a). At this incidence the separated leeside flow structure is evident with the primary body vortex inducing a low pressure region at approximately $\phi=150^{\circ}$ and $x / L>0.6$. In this configuration, the impinging shock strikes the receiver nearside close to the leading edge $\left(x^{\prime} / L=0.09\right)$ and diffracts over the base of the body. As a result, the impinging shock has no influence on the farside flowfield. The predicted nearside impingement location is in good agreement with the measurements and gives $x^{\prime} / L=0.8$.

In spite of the complexity of the flow, there is good overall agreement in qualitative terms between the predicted and measured surface pressure distributions (Fig. 10). However, a small discrepancy exists where the CFD solution predicts that the diffracted shock path exits over the receiver base at a location $\left(\phi \approx 70^{\circ}\right.$, Fig. $\left.10 \mathrm{~b}\right)$ further leeward than the measured results $\left(\phi \approx 20^{\circ}\right.$, Fig. 10 a). Nevertheless, the prediction correctly shows that the diffracted shock does not reach the receiver farside and this is an important characteristic of the interference aerodynamics in this configuration. A comparison of the circumferential pressure distribution towards the aft of the receiver $(x / D=7)$ shows modest agreement between the computations and the measurements (Fig. $10 \mathrm{c}$ ). There is good agreement in 
terms of the first local suction peak $\left(\phi=80^{\circ}\right)$ and subsequent plateau which is associated with the crossflow separation. There is also good agreement between the CFD and the measurements in the location of the second suction peak which is induced by the primary body vortex on the receiver leeside at $\phi=150^{\circ}$ (Fig. $10 \mathrm{c}$ ). However, there is a notable difference in the magnitude of the vortex suction (Fig. $10 \mathrm{c}$ ).

In the equivalent configuration where the receiver is at $\sigma_{R}=15^{\circ}$ and placed alongside the blunt generator the flowfield structures are more intricate. Nevertheless, the predicted surface pressure distribution and characteristics are in fairly good agreement with the PSP measurements (Fig. 11). There is a larger difference between the measured $\left(x^{\prime} / L=0.15\right)$ and prediction $\left(x^{\prime} / L=0.18\right)$ nearside shock impingement locations. Although this difference is non-negligible it is small and most likely due to the predicted location of the local shockinduced separation which begins on the nearside and diffracts around the body. The flow features for this configuration are highly complex. Consequently, this particular configuration is analysed further and in substantial detail in $\S 4.5$ using both the CFD predictions and measurements.

(a)

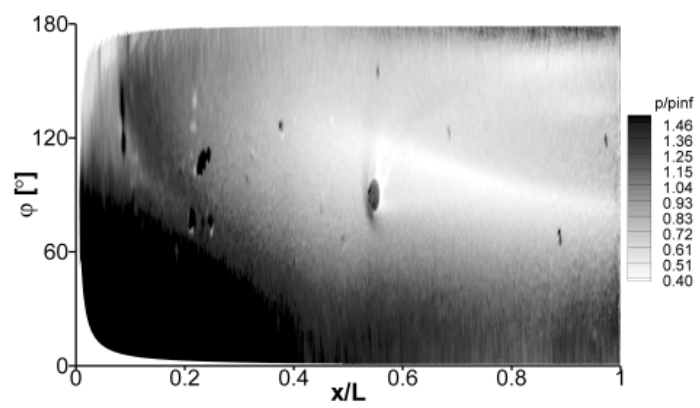

(b)

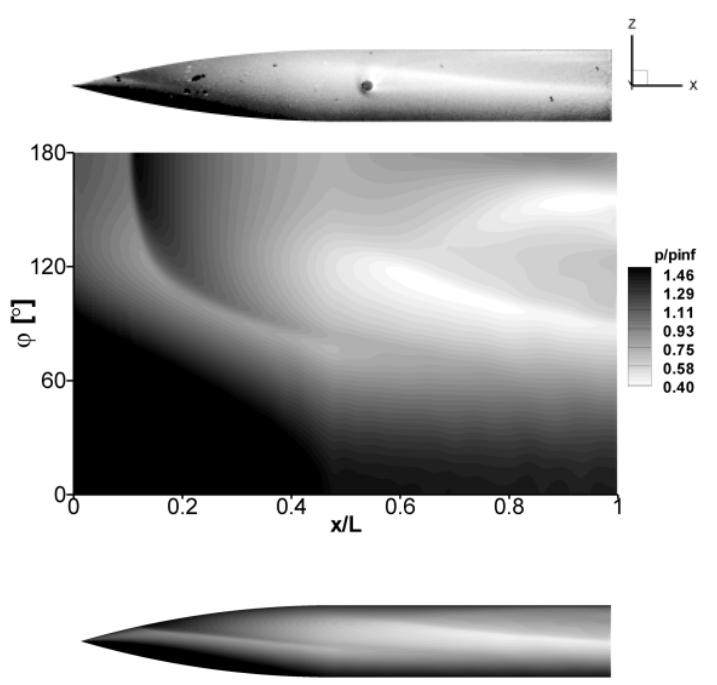


(c)

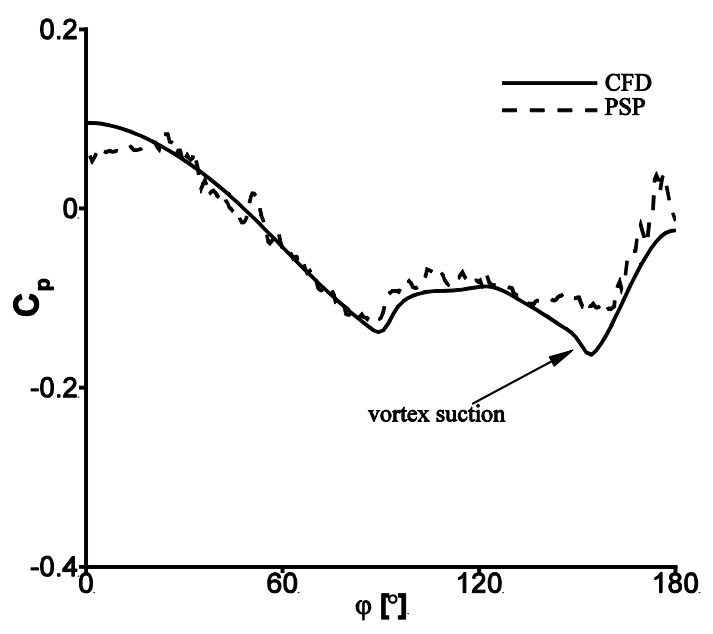

Fig. 10 (a) Measured and (b) predicted surface pressure distributions, (c) quantitative PSP and CFD comparison of circumferential pressure distribution at $x / D=7$ : sharp generator, $\Delta x / D=1.67 \Delta z / D=2.94$, $\sigma_{\mathrm{R}}=15^{\circ} \sigma_{\mathrm{G}}=0^{\circ}$

(a)

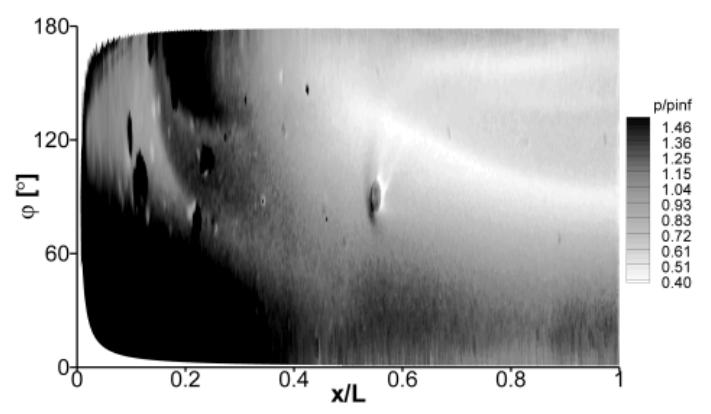

(b)

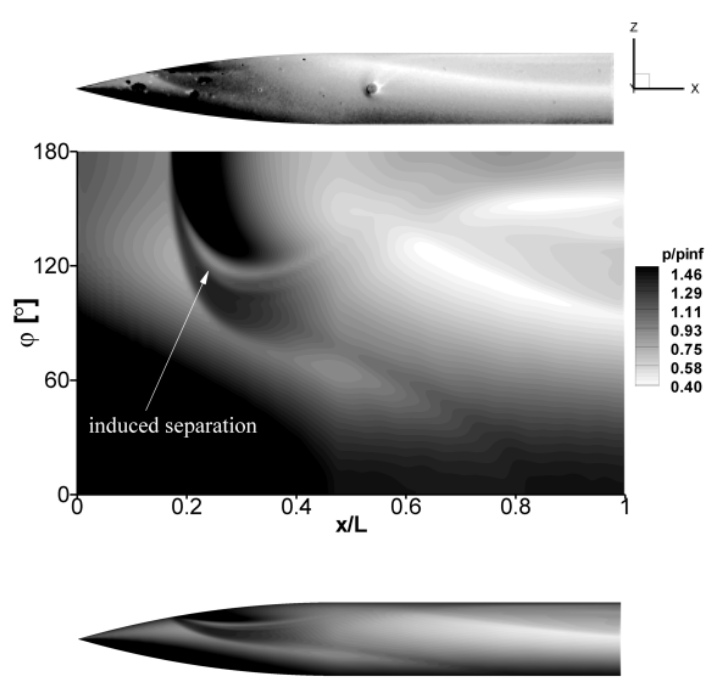


(c)

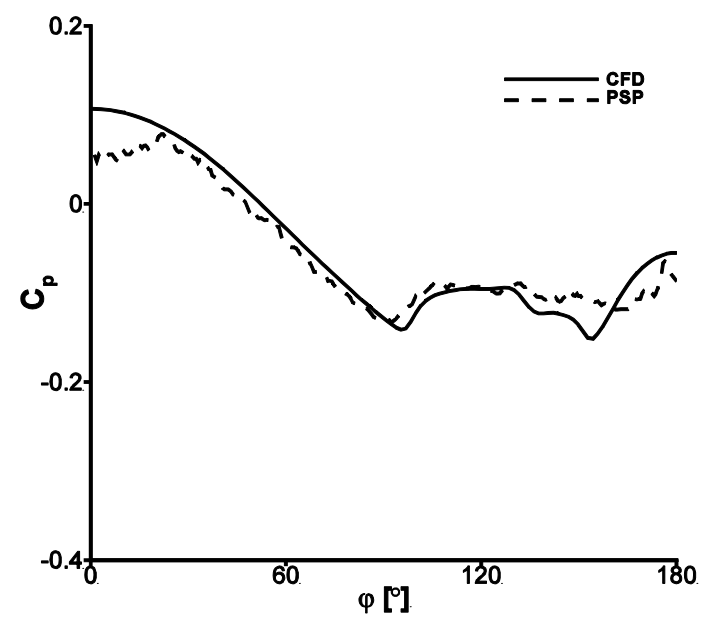

Fig. 11 (a) Measured and (b) predicted surface pressure distributions, (c) quantitative PSP and CFD comparison of circumferential pressure distribution at $x / D=7$ : blunt generator, $\Delta x / D=-0.53 \Delta z / D=2.94$, $\sigma_{\mathrm{R}}=15^{\circ} \sigma_{\mathrm{G}}=0^{\circ}$

Overall, a key selection of the validation dataset is presented above. The comparisons presented are generally representative of the configurations and aerodynamics of the complete dataset. Further validation comparisons are presented in Chaplin [6]. In general, the CFD predictions are in good agreement with the measured receiver surface pressures, interference loads and flowfield visualisations for all configurations. This allows the predicted solutions to be used with confidence in conjunction with the measurements to provide a better understanding of the underlying flow physics. This approach is adopted in the following sections ( $\$ 4.2-4.5$ ) to investigate the effects of axial stagger, receiver incidence and shock induced separation.

\subsection{Effect of axial impingement location}

The previously presented comparisons establish confidence that the CFD method is capable of predicting the detailed interference aerodynamics as well as the overall forces and moments for two bodies in close proximity. Consequently, the CFD predictions are now used in conjunction with the measurements to develop a full and robust understanding of the aerodynamic interference and how it is affected by the axial stagger between the bodies. Previous research into multi-body aerodynamic interference has identified that the axial impingement location of the generator bow shock onto the receiver body is an important parameter $[6,10]$. This primary axial impingement point depends on the axial stagger, lateral separation, receiver incidence and the generator bow shock angle. The effects of receiver incidence $\left(\sigma_{R}\right)$ and axial stagger $(\Delta x)$ are examined here. The configurations considered are for the receiver and sharp generator at five incidence angles $\left(\sigma_{R}=-15,-8,0,8\right.$, $\left.15^{\circ}\right)$ over a range of five axial staggers $(\Delta x / D=-1.65,0,1.67,2.68,3.679)$. The CFD flow solutions are then used in tandem with the experimental evidence to understand the detailed aerodynamics induced by a change in axial impingement location. The effect of axial impingement location is considered in detail for two groups of configurations which capture the range of aerodynamic characteristics for the multi-body arrangements. The first group is when $\sigma_{R}=0^{\circ}(\S 4.3)$ and the second group is for cases where $\sigma_{R}=15^{\circ}(\S 4.4)$.

\subsubsection{Receiver force and moment characteristics}

In general, the measured change in normal force as a result of the interference $\left(\Delta \mathrm{C}_{\mathrm{Z}}\right)$ becomes more negative as the generator (and and hence axial impingement location) move aftward for the majority of the incidence angles presented in Fig. 12a. There is a 
concomitant increase in the pitching moment interference load $\left(\Delta \mathrm{C}_{\mathrm{m}}\right)$, Fig. 12b. However, the cases where the receiver is at a high positive incidence $\left(\sigma_{R}=15^{\circ}\right)$ exhibit a different characteristic. In this case, there is a non-monotonic trend of normal force and pitching moment interference load with axial stagger.

The significance of these results becomes more apparent when the abscissa is changed from the axial stagger parameter $(\Delta x / D)$ to the axial impingement parameter, $x^{\prime} / L$ (Fig. $13 a, b)$. The effect of $x^{\prime} / L$ on the interference loads is generally similar for $-15 \leq \sigma_{R} \leq 8^{\circ}$ and the data points group moderately well together which further underlines the primary importance of this parameter $[6,10]$. The characteristically different results for the configurations at $\sigma_{R}=15^{\circ}$ are further emphasised in Fig. 13a,b. Therefore, representative examples of the different measured trends $\left(\sigma_{R}=0^{\circ}\right.$ and $\left.\sigma_{R}=15^{\circ}\right)$ are further investigated using the predicted flowfield to firstly understand the effect of axial impingement location and secondly to understand why the configurations at $\sigma_{R}=15^{\circ}$ are different.

(a)

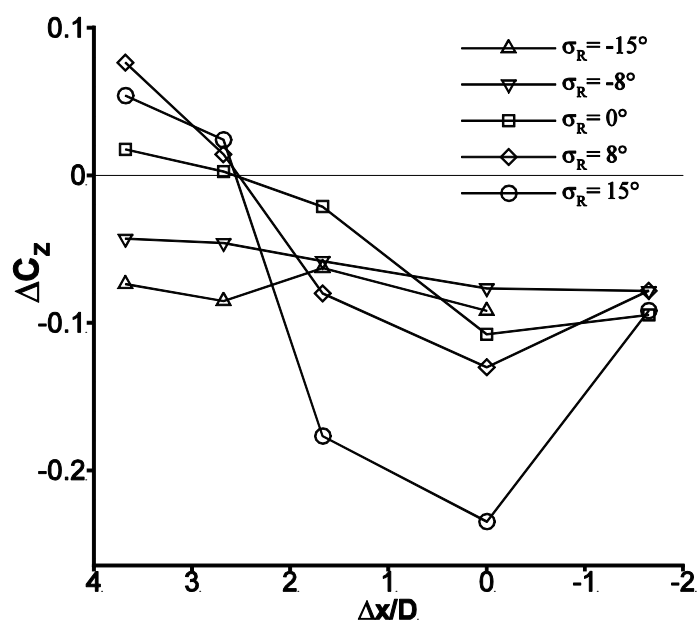

(b)

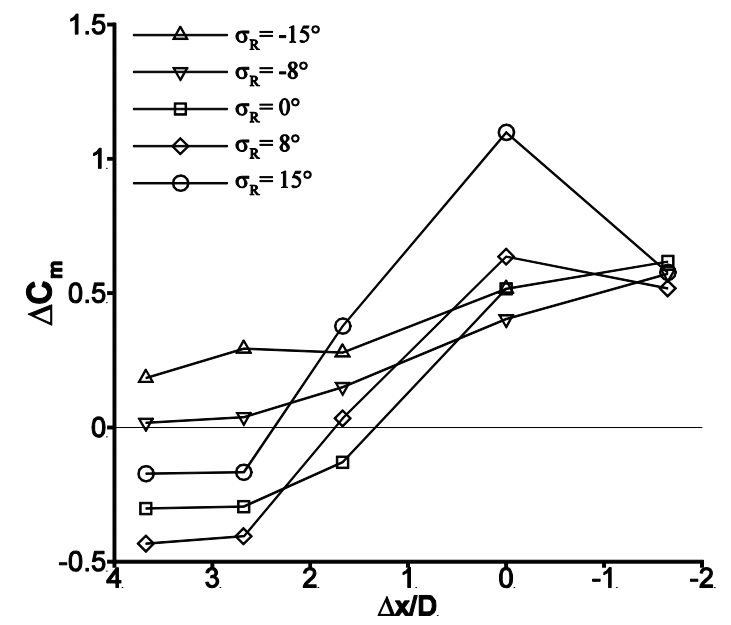

Fig. 12 Effect of axial stagger on measured receiver (a) normal force and (b) pitching moment interference loads: sharp generator, $\Delta z / D=2.94, \sigma_{G}=0^{\circ}$ 
(a)

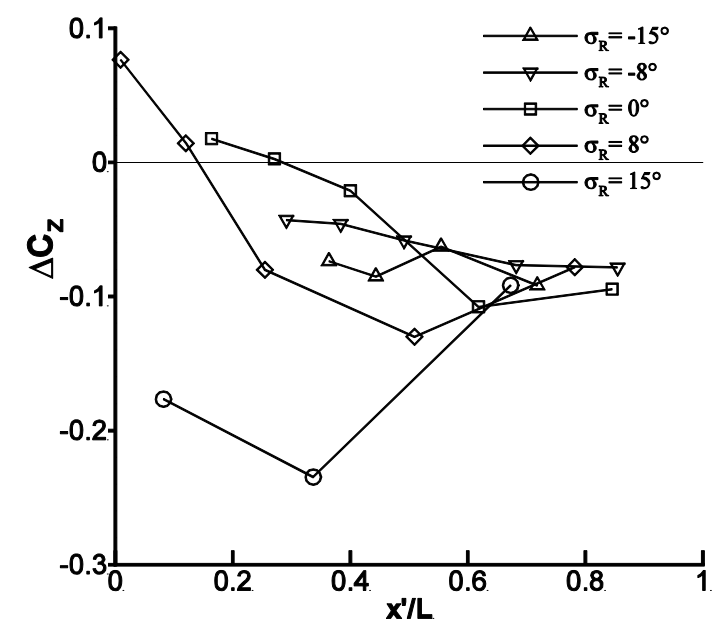

(b)

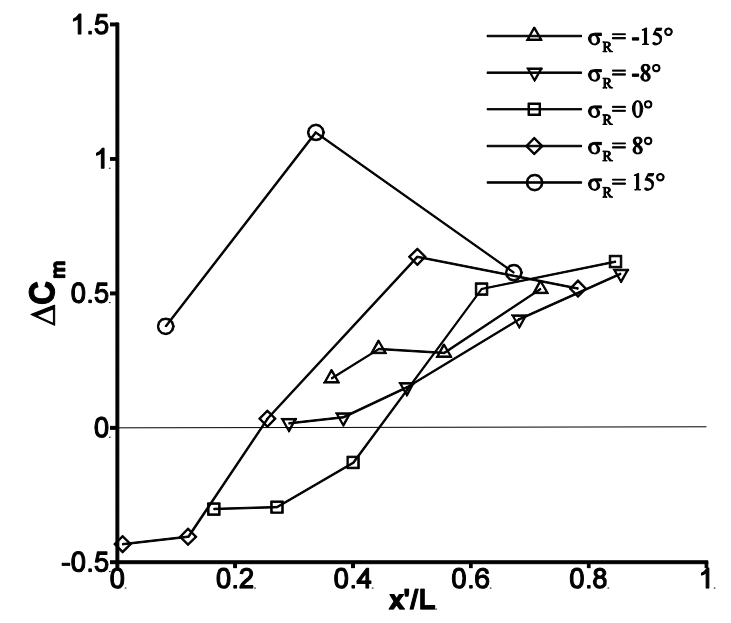

Fig. 13 Effect of axial impingement location on measured (a) normal force and (b) pitching moment interference loads, $\Delta \mathrm{z} / \mathrm{D}=2.94, \sigma_{\mathrm{G}}=0^{\circ}$

\subsection{Effect of axial impingement location for configurations at $\sigma_{\mathrm{R}}=0^{\circ}$}

Over the axial stagger range considered when the receiver is at zero incidence, the movement in the axial impingement point covers $75 \%$ of the receiver body length (Fig. 14 a-

e). As the generator moves from $\Delta x / D=3.679$ upstream of receiver to $\Delta x / D=-1.65$ downstream of receiver, there is a change in the polarity of the induced interference loads (Fig. 15a,b). This is a notable finding because it indicates that the interference effects have a characteristically different impact on the receiver forces and moments depending almost entirely on the axial impingement location. Similar results were also found in previous studies $[3,4,9]$. When the impingement location is near the leading edge (Fig. 14a) there is a positive normal force interference force $\left(\Delta C_{z}\right)$ which attracts the receiver towards the generator body. However, this changes to a repulsive force when the shock impinges near the trailing edge (Fig. 14e, Fig. 15a). Although there is a mutual attractive force when the primary shock impingement point $\left(x^{\prime} / L\right)$ is near the leading edge (Fig. $14 \mathrm{a}$ ), there is a strong induced pitch-away moment $\left(\Delta \mathrm{C}_{\mathrm{m}}<0\right.$, Fig. $\left.15 \mathrm{~b}\right)$. This initially benign pitch-away moment changes polarity to a nose-up induced $\Delta \mathrm{C}_{\mathrm{m}}>0$ as $\mathrm{x}^{\prime} / \mathrm{L}$ occurs towards the trailing edge. Overall, there is fairly good agreement between the measured and predicted interference loads and the flowfield CFD solutions are now used to understand why the observed change in polarity occurs for the receiver body at zero incidence. 
(a) $\Delta x / D=3.679, x^{\prime} / L=0.16$

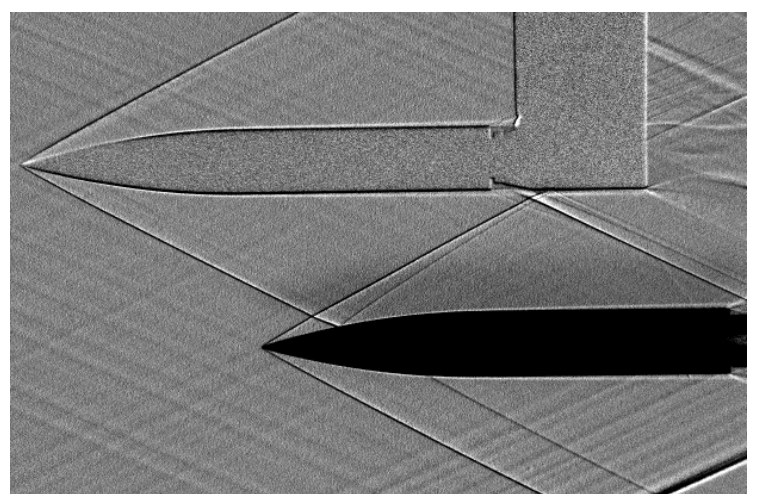

(b) $\Delta x / D=2.68, x^{\prime} / L=0.27$
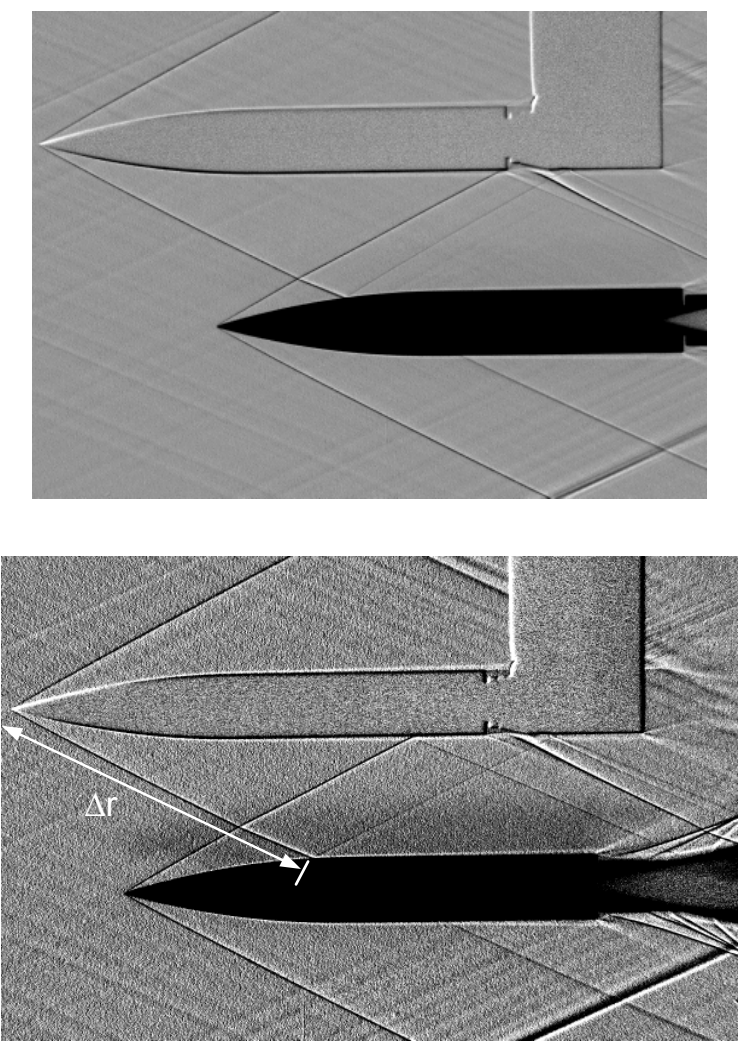

(c) $\Delta x / D=1.67, x^{\prime} / L=0.4$

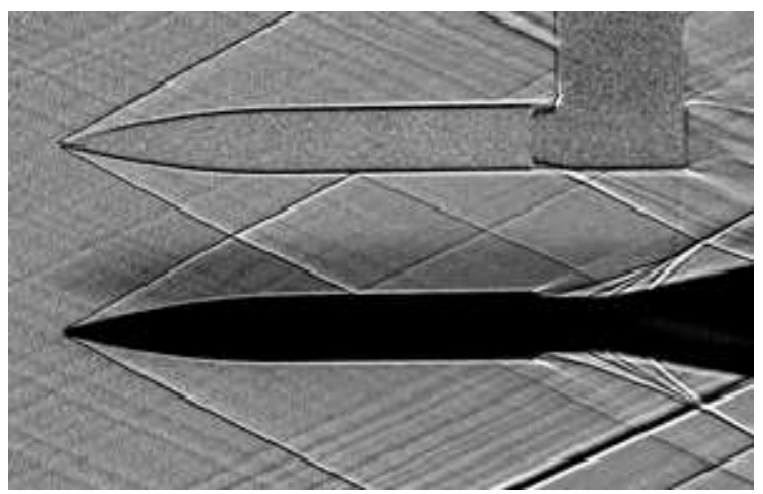

(d) $\Delta x / D=0, x^{\prime} / L=0.62$ 
(e) $\Delta x / D=-1.65, x^{\prime} / L=0.85$

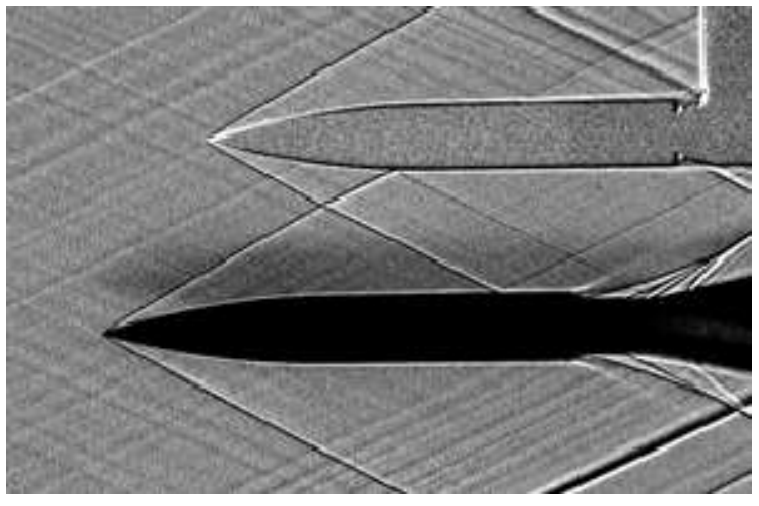

Fig. 14 Measured shadowgraph visualisations for different axial stagger settings (a-e): sharp generator, $\Delta z / D=2.94, \sigma_{R}=0^{\circ} \sigma_{G}=0^{\circ}$

(a)

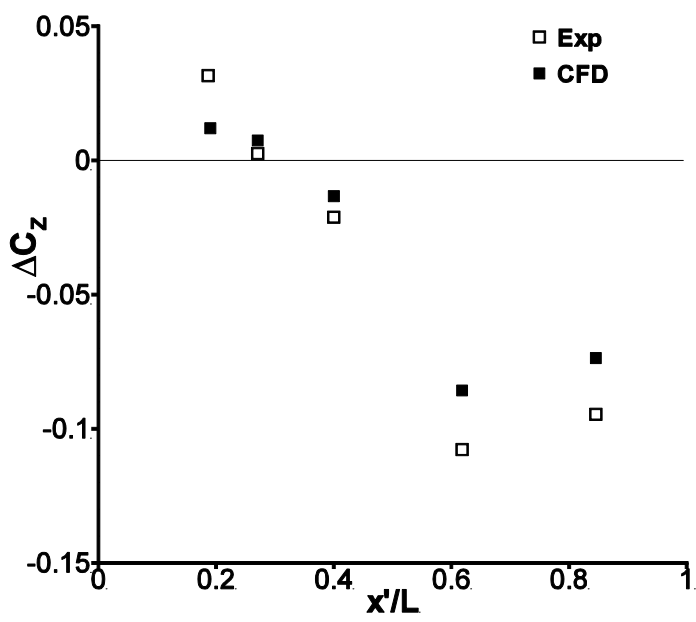

(b)

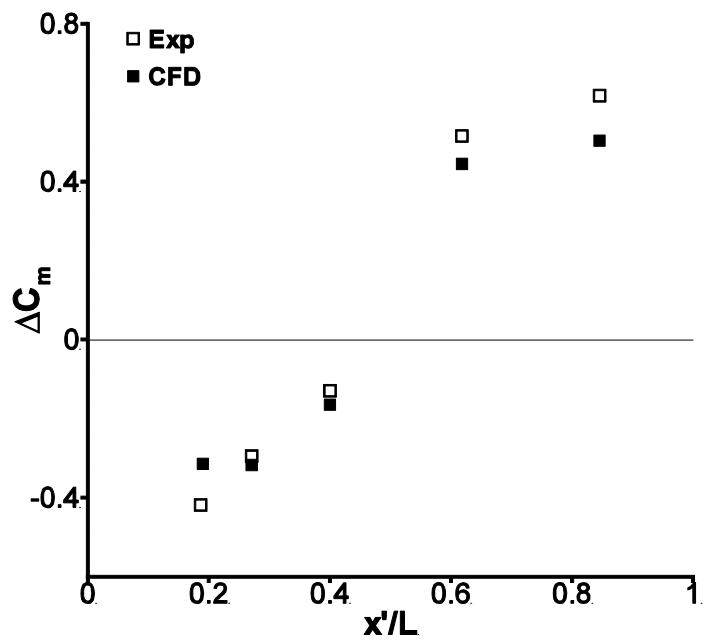

Fig. 15 Effect of axial impingement location on receiver (a) normal force and (b) pitching moment interference loads: sharp generator, $\Delta z / D=2.94, \sigma_{R}=0^{\circ} \sigma_{G}=0^{\circ}$ (uncertainty bars omitted)

\subsubsection{Flowfield mechanisms for the configurations at $\sigma_{R}=0^{\circ}$}

In all five axial stagger configurations where the receiver is at zero incidence (Fig. 14), a portion of the impinging shock from the sharp generator diffracts around the receiver body. The diffracted wavefront reaches the receiver farside for all cases except for $\Delta x / D=-1.65$ (Fig. $14 \mathrm{e}$ ) where it crosses the body centreline $\left(\phi \approx 90^{\circ}\right)$ at the trailing edge of the body. Since the lateral separation is fixed, the distance from the generator leading edge to the 
impingement location ( $\Delta \mathrm{r} / \mathrm{D}$, Fig. $14 \mathrm{c}$ ) is approximately the same for each axial stagger configuration. As a result, the local pressure rise on the receiver nearside $\left(\phi=180^{\circ}\right)$ induced by the impinging shock remains approximately constant at $\Delta \mathrm{C}_{\mathrm{p}, \text { near }} \approx 0.1$ (Fig. 16) for this range of axial stagger configurations.

After the initial nearside pressure rise, there is a subsequent decrease in pressure as a result of the expansion wave field for all configurations (Fig. 16). The axial impingement location has a notable influence on the impact of the expansion wave field. When $x^{\prime} / L$ is near the leading edge the resultant local expansion wave field which acts on the receiver nearside is a combination of the both the impinging expansion waves which emanate from the generator forebody as well as the underlying expansion flow on the receiver forebody. This results in a strong negative pressure gradient in the forebody region immediately after the initial impinging shock pressure rise for the $\Delta x / D=3.679$ and $\Delta x / D=2.68$ cases (Fig. 16). Conversely, a more moderate negative pressure gradient is observed on the receiver nearside when $x^{\prime} / L$ is further aft $(\Delta x / D=1.67, \Delta x / D=0$ and $\Delta x / D=-1.65$ in Fig. $14 \mathrm{c}-\mathrm{e})$. In these cases the local flow is only affected by the impinging expansion waves from the generator forebody (Fig. 16). These subtle differences are important because the extent of the regions of nearside differential pressure are strongly affected by how quickly the local pressure reduces after the initial rise due to the impinging shock.

The effect of the disturbance flowfield is also observed on the receiver farside and this has a key role in the impact on the overall changes in the forces and moments. The impinging shock splits into two diffracting shocks on either side of the receiver. As these diffracting shock legs reach the farside, they cross the body centreline and superimpose to increase the local static pressure (Fig. 17). The farside pressure distributions for each of the different axial stagger configurations are very similar and show a modest initial rise due to the diffracted shock followed by a modest reduction in the local pressure. It should also be noted that although the shocks diffract to the receiver farside, the diffracted expansion waves do not have the same impact on the local farside flowfield i.e. the local farside pressure is never reduced to a level below the isolated configuration (Fig. 17). This is an important aspect because it means that all of the farside interactions contribute solely to a positive change in normal force since $\Delta \mathrm{C}_{\mathrm{p}}>0$.

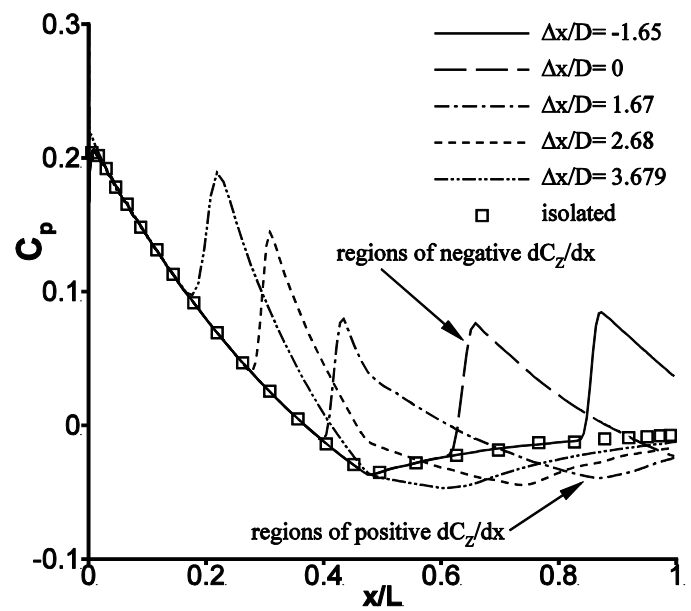

Fig. 16 Effect of axial stagger on predicted receiver nearside pressure distribution $\left(\varphi=180^{\circ}\right)$ : sharp generator, $\Delta z / D=2.94 \sigma_{R}=0^{\circ} \sigma_{G}=0^{\circ}$ 


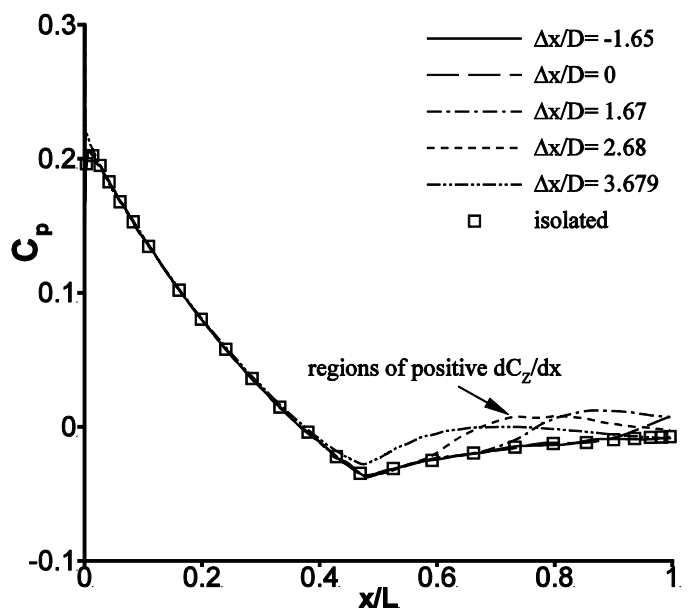

Fig. 17 Effect of axial stagger on predicted receiver farside pressure distribution $\left(\varphi=0^{\circ}\right)::$ sharp generator, $\Delta z / D=2.94, \sigma_{R}=0^{\circ} \sigma_{G}=0^{\circ}$

The local normal force distribution $\left(\mathrm{dC}_{\mathrm{Z}} / \mathrm{dx}\right)$ which acts on the receiver body is strongly influenced by the axial impingement location. The $\mathrm{dC}_{\mathrm{Z}} / \mathrm{dx}$ is a function of the magnitude of the induced differential pressure regions as well as the projected area over which these regions act (Fig. 18). In the current configurations at $\sigma_{R}=0^{\circ}$, there are three important factors which contribute to the local force distribution. The primary shockwave induces an increase in static pressure on the receiver nearside and will deliver a reduction in $C_{z}\left(\Delta d C_{z} / d x<0\right.$, Fig. 18). Conversely, there are regions caused by the expansion waves where the induced nearside differential pressure is negative and there is an associated increase in $\mathrm{C}_{Z}$ $\left(\Delta \mathrm{dC}_{\mathrm{Z}} / \mathrm{dx}>0\right.$, Fig. 18). Finally, on the receiver farside the induced change in static pressure contributes to a positive change in $C_{Z}\left(\Delta d C_{Z} / d x>0\right.$, Fig. 18).

When the axial impingement location is near the leading edge, there is a positive overall $\Delta \mathrm{C}_{z}$ which is the result of the dominance of the impinging expansion waves on the receiver nearside and the farside influence of the diffracted shock (Fig. 14 a). As the axial impingement point moves further aft, the extent of these regions of differential pressure which contribute to a positive local normal force diminish. As a result, the overall $\Delta \mathrm{C}_{z}$ reduces as the $\Delta \mathrm{dC} / \mathrm{dx}<0$ region becomes a larger portion of the overall interference load. Moreover, because the nearside region of positive differential pressure occurs further aft along the body as $x^{\prime} / L$ increases, the moment arm increases for a similar magnitude of local normal force. This effect, coupled with the reduction in the $\Delta \mathrm{dC}_{\mathrm{Z}} / \mathrm{dx}>0$ regions, leads to an increase in $\Delta \mathrm{C}_{\mathrm{m}}$. 


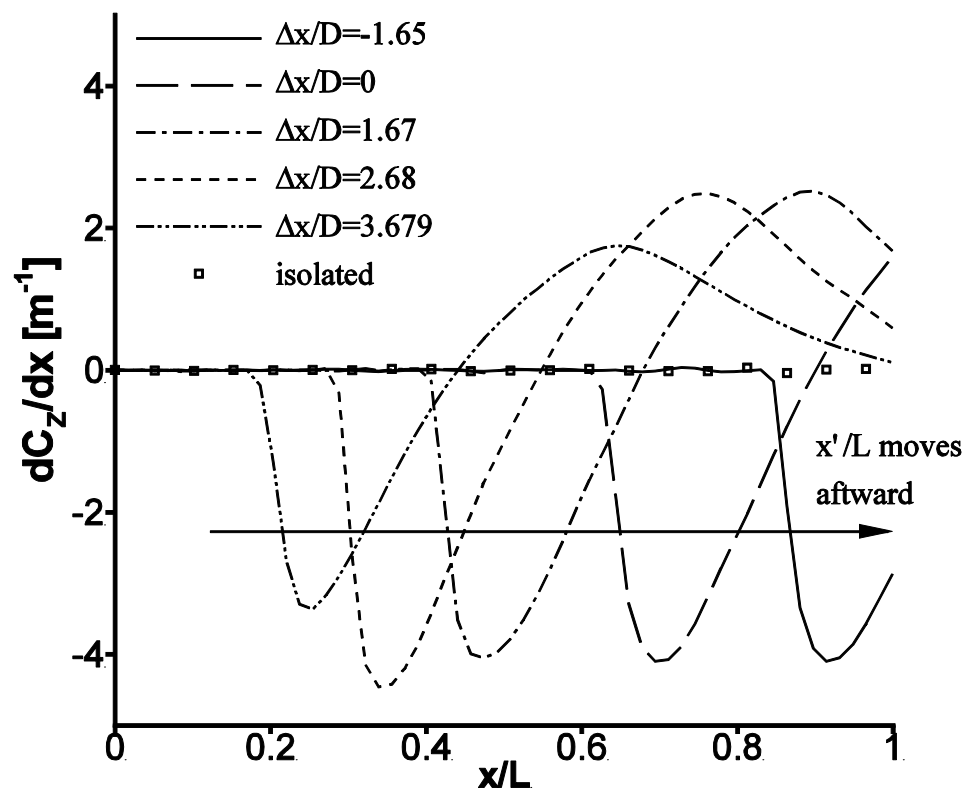

Fig. 18 Effect of axial stagger on predicted receiver local normal force distribution: sharp generator, $\Delta z / D=2.94, \sigma_{R}=0^{\circ} \sigma_{G}=0^{\circ}$

\subsection{Effect of axial impingement location for configurations at $\sigma_{R}=15^{\circ}$}

It is clear from Fig. 13a,b, that the configurations where the receiver incidence is $\sigma_{R}=15^{\circ}$ are characteristically different from the other cases. The purpose of this section is to use the combination of experimental and computational data to understand the detailed aerodynamics and the reason for this difference.

When the receiver incidence is $\sigma_{R}=15^{\circ}$ and an axial stagger of $\Delta x / D=2.68$ is imposed, the generator bow shock passes sufficiently close to the receiver leading edge to influence the receiver flowfield (Fig. 19 a). For the other axial configurations (Fig. 19 b-d) the generator bow shock impinges on the receiver nearside (and leeside) flow structure. However, unlike the cases with the receiver at a lower incidence (Fig. 14), the diffracted shock is not visible on the farside of the receiver body.

Overall, a non-monotonic trend of interference load as a function of axial impingement location is observed in Fig. $20 \mathrm{a}$ and b. Moreover, there is good agreement between the measured and predicted forces and moments and the CFD flowfield solutions are used to evaluate the detailed aerodynamics and understand the effect of axial impingement location.

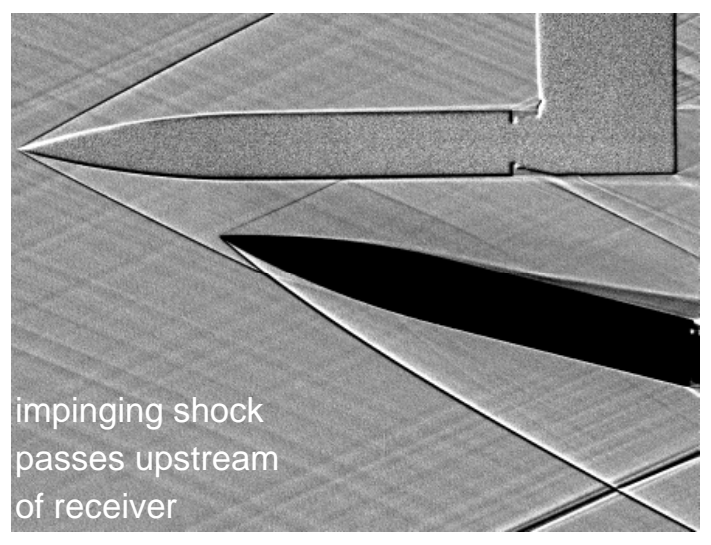


(a) $\Delta x / D=2.68$

(b) $\Delta x / D=1.67, x^{\prime} / L=0.08$
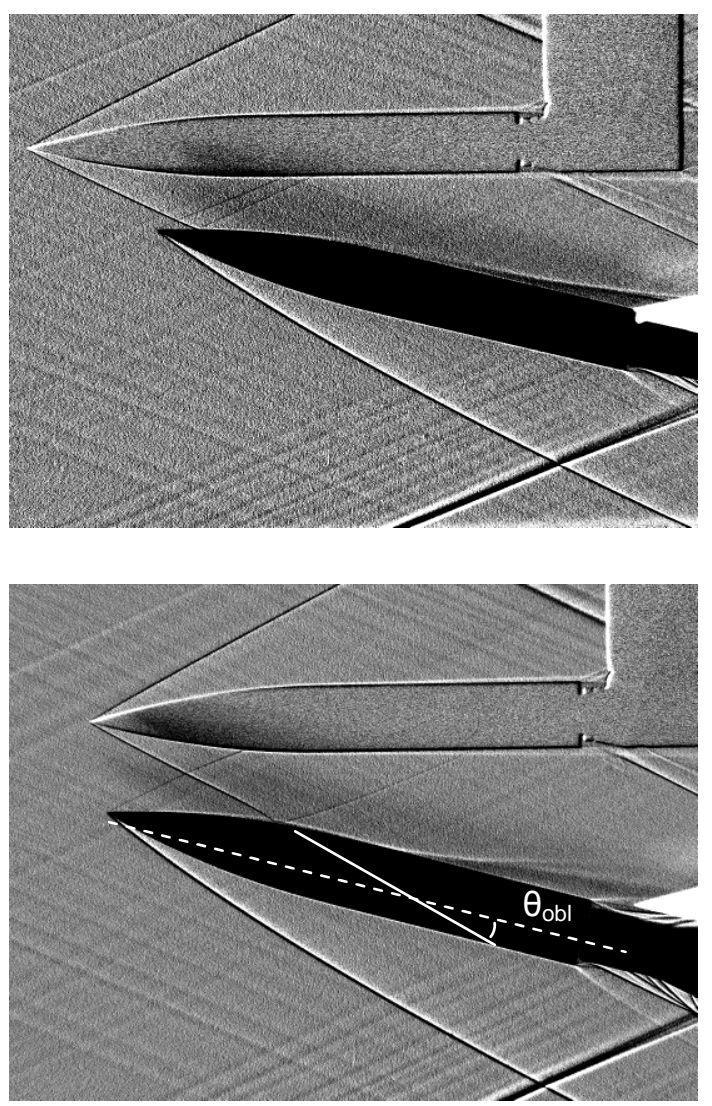

(c) $\Delta x / D=0, x^{\prime} / L=0.34$

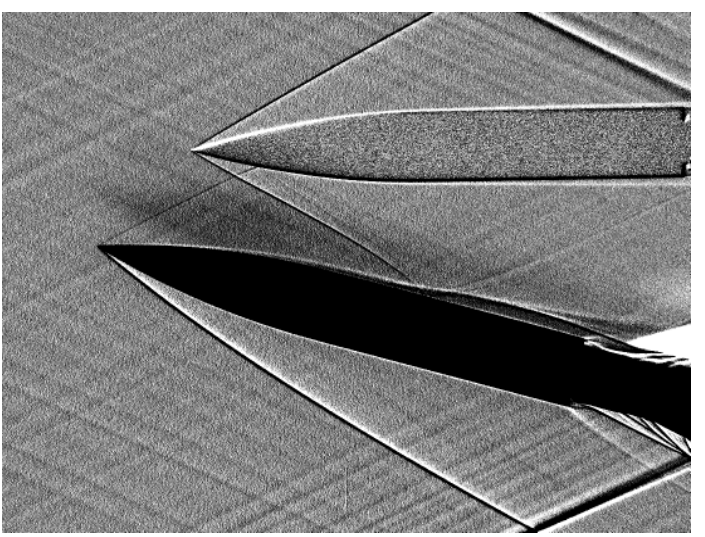

(d) $\Delta x / D=-1.65, x^{\prime} / L=0.67$

Fig. 19 Measured shadowgraph visualisations for different axial stagger settings (a-d) sharp generator, $\Delta z / D=2.94, \sigma_{R}=15^{\circ} \sigma_{G}=0^{\circ}$ 
(a)

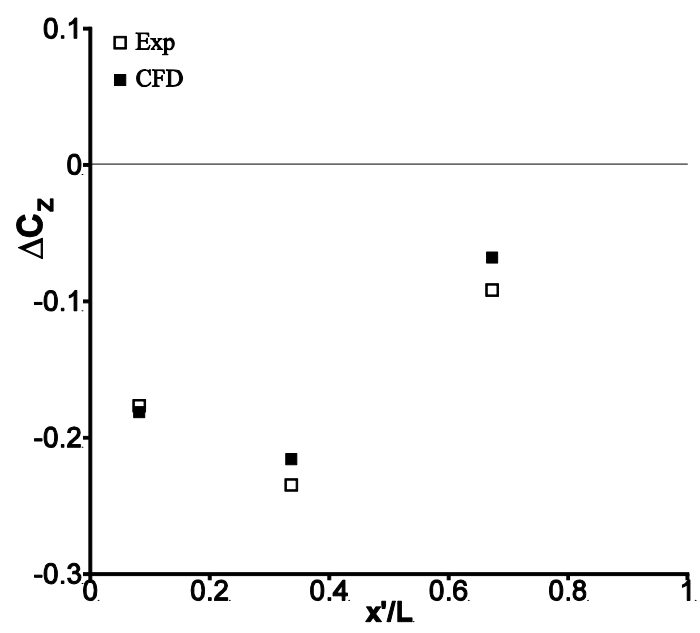

(b)

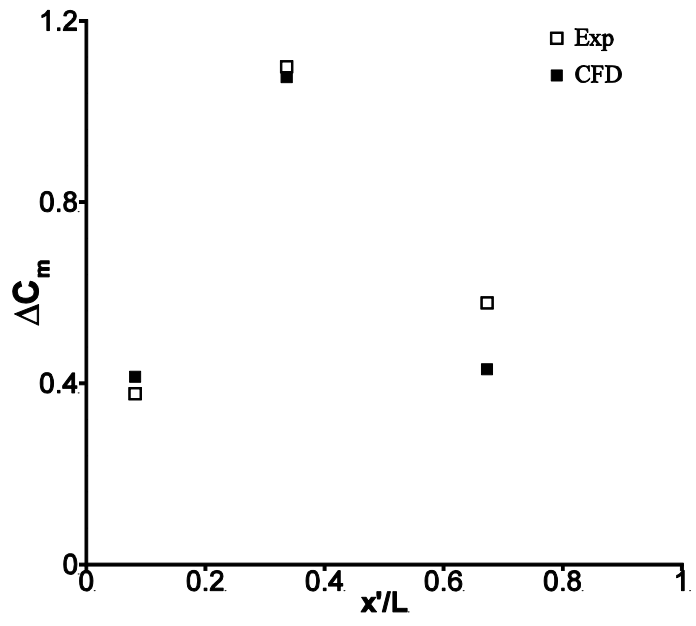

Fig. 20 Effect of axial impingement location on receiver (a) normal force and (b) pitching moment interference loads: sharp generator, $\Delta z / D=2.94, \sigma_{R}=15^{\circ} \sigma_{G}=0^{\circ}$

In comparison to the configurations at $\sigma_{\mathrm{R}}=0^{\circ}$, one of the main differences for these four axial stagger configurations is that the primary impinging shock does not diffract to the farside of the receiver (Fig. 19). In the three configurations where the generator bow shock impinges on the receiver, the shock obliqueness angle $\left(\theta_{\mathrm{obl}} \approx 13^{\circ}\right.$, shown in Fig. $\left.19 \mathrm{c}\right)$ and geometric arrangement are such that the diffracted shock passes over the base of the receiver and does not reach the farside (Fig. 10 a, Fig. 21 and Fig. 22). This observation is confirmed with examination of the farside pressure distributions where there is no influence of the diffracted shock on the isolated values for all axial configurations (Fig. 23). Consequently, the effect of axial impingement location on the interference loads is primarily determined by the nature of the interactions between the impinging disturbances and the receiver nearside flowfield (Fig. 24).

4.4.1 Flowfield mechanisms for the configurations at $\sigma_{R}=15^{\circ}$

A nearside pressure rise is observed for all axial stagger configurations as shown in Fig. 24. This is caused by the impinging shock for the configuration where $\Delta x / D=-1.65,0,1.67$. For the $\Delta x / D=2.68$ configuration it is the result of the compression from the generator bow shock which passes upstream of the receiver leading edge. The characteristics of the nearside interactions for the two configurations where the generator is upstream of the receiver $(\Delta x / D=2.68$ and $\Delta x / D=1.67)$ are different from the other two configurations. As a result, the characteristics of the configuration where the generator is axially aligned $(\Delta x / D=0)$ and downstream of the receiver $(\Delta x / D=-1.65)$ are now discussed in more detail. 
When the receiver is at this relatively high incidence of $\sigma_{R}=15^{\circ}$, the underlying aerodynamics of the isolated slender body are strongly affected by the formation of the body vortices. For a slender body of this fineness ratio $(L / D=7.358)$, these vortices begin to occur when the incidence is typically greater than $\sigma_{R}=8^{\circ}$. The vortices are associated with the crossflow boundary-layer separation which leads to the vortex roll-up and is characterised by a clear pattern of surface separation and reattachment lines. The vortices have a strong core axial velocity and consequently they produce a notable region of low pressure, additional surface suction and hence contribute to the body normal force and pitching moment. The formation of these vortices is affected by the impinging disturbance flowfield. In particular, when the axial location of the impinging shock is upstream of the crossflow separation associated with the body vortices (as for $\Delta x / D=2.68$ and $\Delta x / D=1.67$ ), the expansion waves from the generator forebody strongly reduce the receiver nearside pressure after the initial rise. Moreover, the impinging shock turns the flow in the opposite direction to the natural crossflow induced by the body incidence. This has the effect of delaying the axial location of the primary vortices to further aft along the receiver body in comparison to the isolated configuration (Fig. $25 \mathrm{a}-\mathrm{c}$ ). Evidence of this can be seen in the corresponding nearside pressure distributions for both configurations in Fig. 24 where the pressure footprints show that the development of the body vortices occur further aft on the body.

When the shock impingement location is aft of the crossflow separation location (as in $\Delta x / D=0$ and $\Delta x / D=-1.65$ ) the impinging expansion waves have little effect on the nearside flowfield and the body vortex is the dominant flow feature in determining the leeside pressure distribution (Fig. 24) after the initial compression from the impinging shock. For example, in the $\Delta x / D=0$ case the local pressure trend after the initial rise is similar to the isolated configuration where the body vortices dominate the local pressure distribution. The predicted skin friction line plots for these configurations (Fig. 25 a,d-e) show there is little effect of the interference flowfield on the vortex location since the shock impingement point is aft of the crossflow separation location for the isolated body configuration.

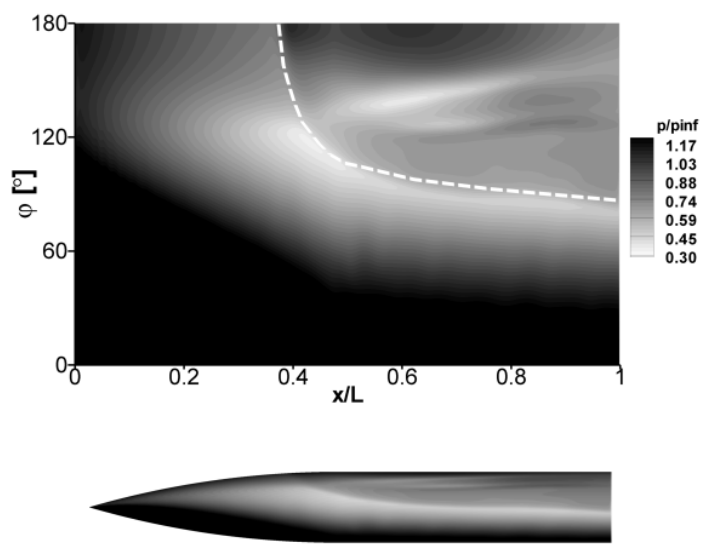

Fig. 21 Predicted receiver surface pressure contours highlighting approximate impinging shock path (dashed): sharp generator, $\Delta \mathrm{x} / \mathrm{D}=0 \Delta \mathrm{z} / \mathrm{D}=2.94, \sigma_{\mathrm{R}}=15^{\circ} \sigma_{\mathrm{G}}=0^{\circ}$ 


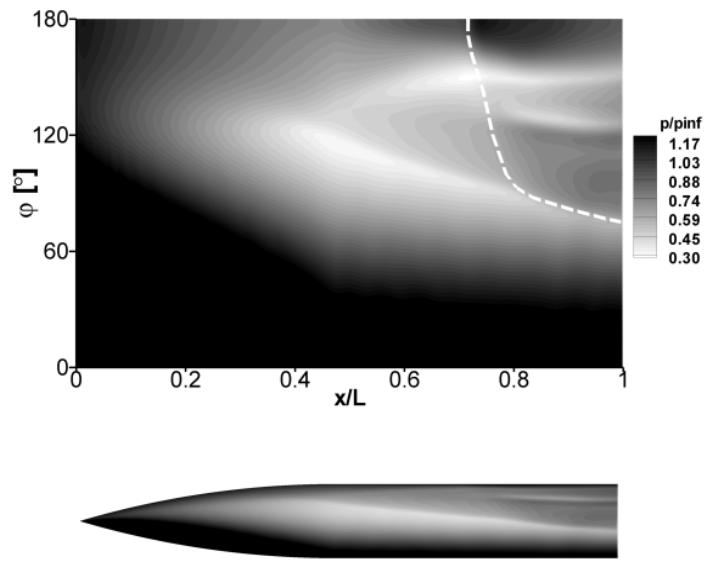

Fig. 22 Predicted receiver surface pressure contours highlighting approximate impinging shock path (dashed): sharp generator, $\Delta x / D=-1.65 \Delta z / D=2.94, \sigma_{R}=15^{\circ} \sigma_{G}=0^{\circ}$

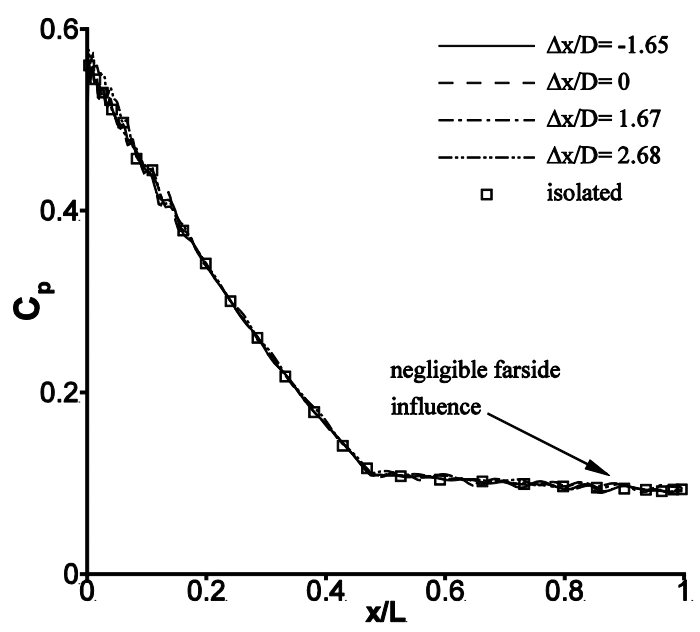

Fig. 23 Effect of axial stagger on predicted receiver farside pressure distribution $\left(\phi=0^{\circ}\right)$ : sharp generator, $\Delta z / D=2.94 \sigma_{R}=15^{\circ} \sigma_{G}=0^{\circ}$.

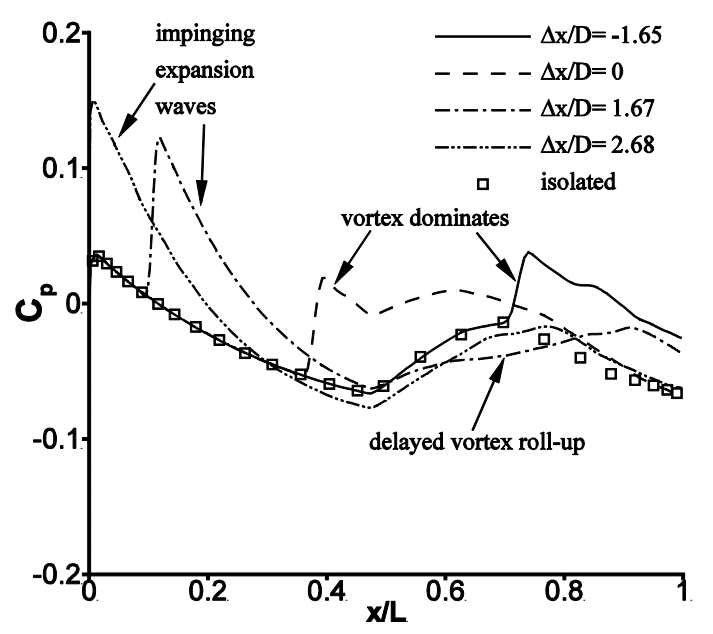

Fig. 24 Effect of axial stagger on predicted receiver nearside pressure distribution $\left(\phi=180^{\circ}\right)$ : sharp generator, $\Delta z / D=2.94 \sigma_{R}=15^{\circ} \sigma_{G}=0^{\circ}$. 


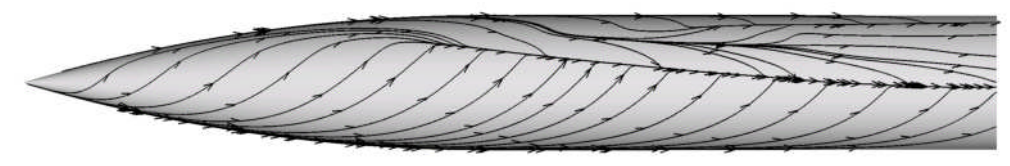

(a) isolated $\sigma_{R}=15^{\circ}$

(b) $\Delta x / D=2.68$

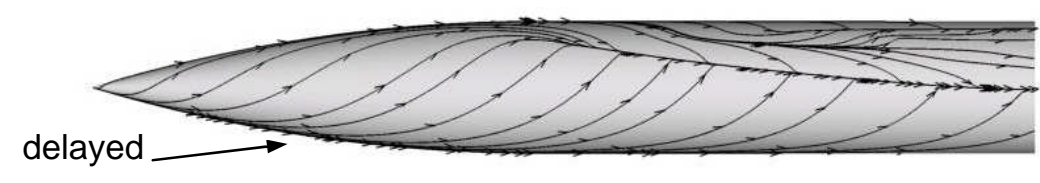

vortex

roll-up

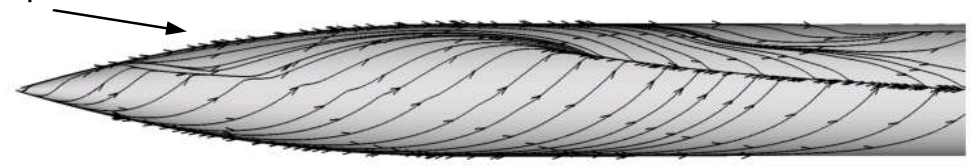

(c) $\Delta x / D=1.67$

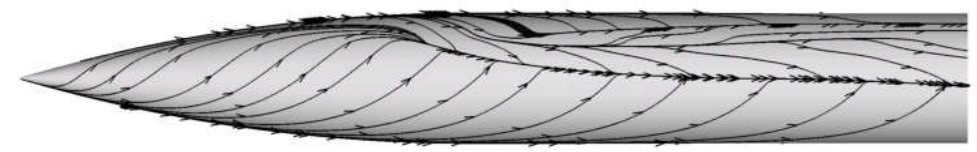

(d) $\Delta x / D=0$

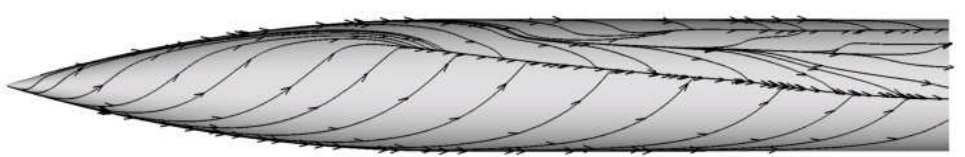

(e) $\Delta x / D=-1.65$

Fig. 25 Predicted surface skin friction vector lines for the (a) isolated configuration and (b-e) different axial stagger settings: $\Delta \mathrm{z} / \mathrm{D}=2.94, \sigma_{\mathrm{R}}=15^{\circ} \sigma_{\mathrm{G}}=0^{\circ}$

\subsubsection{Receiver local normal force distributions for configurations at $\sigma_{R}=15^{\circ}$}

The axial impingement location has a strong effect on the receiver nearside pressure distribution and the regions of differential pressure, in turn, affect the local normal force distribution $\left(\mathrm{dC}_{\mathrm{z}} / \mathrm{dx}\right.$, Fig. 26). This can also be further examined by expressing $\mathrm{dC}_{\mathrm{Z}} / \mathrm{dx}$ as a change in the local normal force distribution from the isolated configuration $\left(\Delta d C_{Z} / d x\right)$ in Fig. 27 in order to visualise the interference characteristics more clearly. When the generator bow shock misses the receiver $(\Delta x / D=2.68)$ the local normal force distribution is dominated by the positive local normal force region induced by the impinging expansion waves which act over a large portion of the body nearside. This leads to an overall positive normal force interference load $\left(\Delta C_{Z}=0.03\right)$ and a negative pitching moment interference load $\left(\Delta C_{m}=-0.17\right)$. As the impingement location moves aft, the influence of the impinging expansion waves diminishes because they act over a smaller extent until they have a negligible effect as in the $\Delta x / D=0$ and $\Delta x / D=-1.65$ cases. For the latter two configurations, there are no regions of positive $\Delta \mathrm{dC}_{\mathrm{Z}} / \mathrm{dx}$ and the interference footprints contribute solely to a negative overall normal force interference load (Fig. 27). Finally, the $\Delta x / D=1.67$ and $\Delta x / D=0$ configurations induce the largest magnitude of interference loads (Fig. 20a and b) as a result of the large extent over which the nearside region of positive differential pressure acts on the body (Fig. 27). 


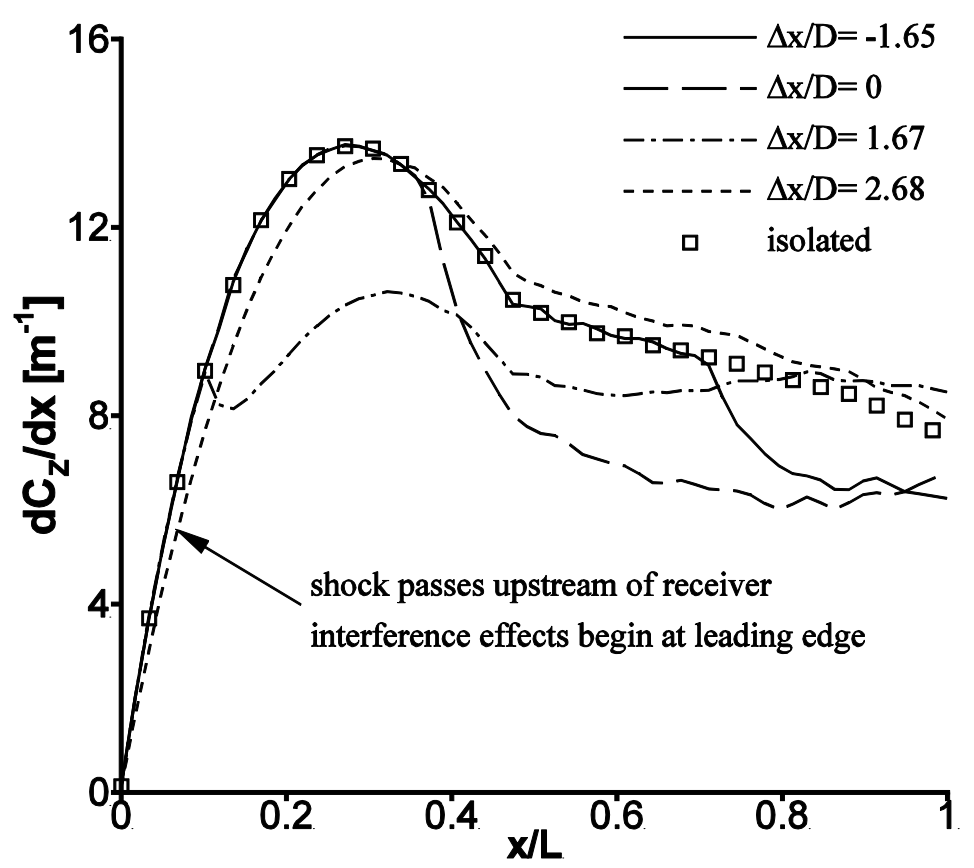

Fig. 26 Effect of axial stagger on predicted receiver local normal force distribution: sharp generator, $\Delta \mathrm{z} / \mathrm{D}=2.94, \sigma_{\mathrm{R}}=15^{\circ} \sigma_{\mathrm{G}}=0^{\circ}$.

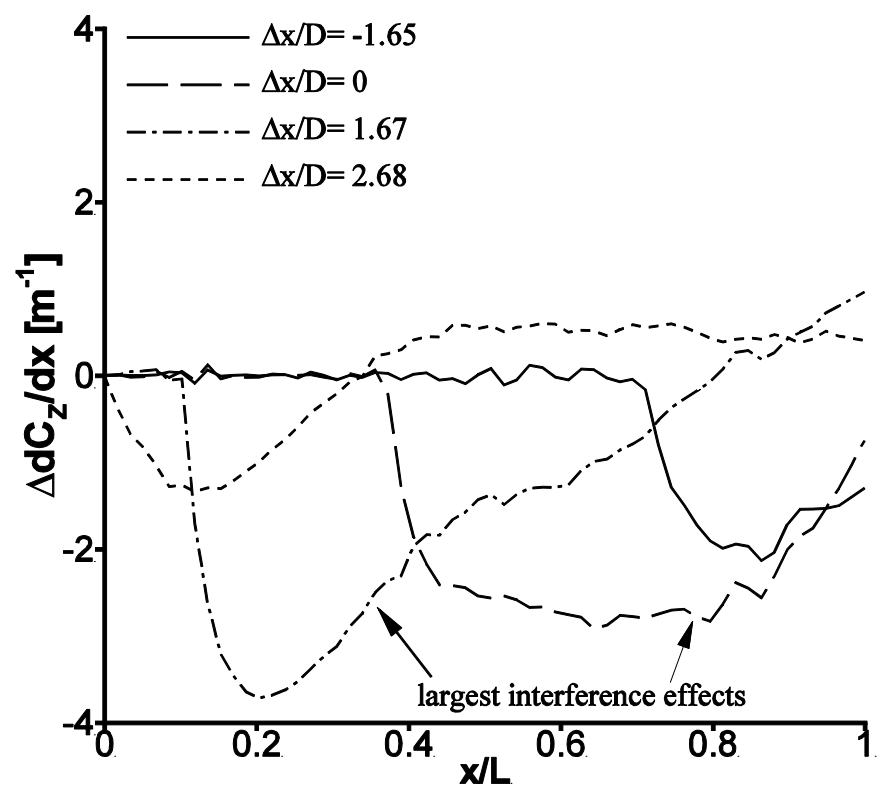

Fig. 27 Effect of axial stagger on predicted receiver change in local normal force distribution from the isolated configuration: sharp generator, $\Delta z / D=2.94, \sigma_{R}=15^{\circ} \sigma_{G}=0^{\circ}$.

\subsection{Strong viscous interaction}

The discussion thus far, has shown that the impinging shock can change the characteristics of the viscous flow features such as the body vortices and boundary-layer and that such viscous flow features are important aspects of the receiver body flowfield. The importance of these viscous flow features are expected due to their notable impact on the surface pressure distribution and thus the body loads for a slender body at high incidence (\$4.4). This is further examined for a configuration which exhibits a strong interaction with the viscous boundary-layer and the combination of measured data and CFD predictions are used to understand and characterise the flow physics. 
The effect and sensitivity of the skewed boundary-layer and the body vortices increases with body incidence. For a particular configuration with a blunt generator $\left(\Delta x / D=-0.53, \sigma_{R}=15^{\circ}\right)$ the impinging generator bow shock induces a large nearside pressure rise on the receiver body $\left(\Delta \mathrm{C}_{\mathrm{p}, \text { near }}=0.47\right)$ as a result of the initial shock strength and the relative close proximity between the impingement location and the generator leading edge. This results in a shock induced boundary-layer separation on the receiver nearside. The evidence for this is in the shadowgraph visualisation (Fig. 28), which shows a double shock reflection from the receiver nearside which is a typical characteristic of a shock induced separation. The double reflected shock structure is due to the pre-compression and re-attachment shocks on the receiver nearside and a similar flowfield feature was also observed for a shock impinging on a cylindrical body by Brosh and Kussoy [25] and Morkovin [17]. An additional notable difference is that the diffracted shock on the leeward side of the receiver also has a double shock structure

In addition to the pressure-driven shock induced separation, the induced crossflow associated with the impinging shock turns the flow in the windward direction (Fig. 29 a). This is in opposition to the natural crossflow over the body, which occurs as a result of the receiver incidence and leads to a local induced crossflow separation region. The importance of induced crossflow was also observed in similar studies of a shock impinging on a cylindrical body by Brosh [7, 25] and Morkovin et al. [15].

\subsubsection{Horse-shoe vortex system}

As a result of the diffracted shock path, the local separation region generates one half of a horse-shoe vortex pair on either side of the body (Fig. 28, Fig. 29 a). A well defined separation line, $\mathrm{S} 1$ line is predicted (Fig. 29 b). A vortex sheet rolls-up into a small horseshoe vortex structure with its core close to the surface and the flow re-attaches further leeward at A2. There is also evidence of a weaker secondary vortex underneath the horseshoe vortex with the secondary separation and attachment lines S2 and A3 respectively.

The horse-shoe vortex structure initially extends windward in the direction of the shock path with increasing $x / L$. After a short distance along the body, the momentum of the natural crossflow begins to dominate the local flow structure and drives the horse-shoe vortex in the leeward direction. It reaches close to the symmetry plane and then travels towards the base of the receiver as shown by the streamtrace ribbons in Fig. 29 a. This is notable because the horseshoe vortex generates a low pressure region underneath the core as can be seen in Fig. 29 b. This has a moderate vortex suction effect on the local pressure distribution and is a direct consequence of the upstream viscous interaction. Further evidence of this additional horseshoe vortex is seen in the shadowgraph visualisation in Fig. 28 


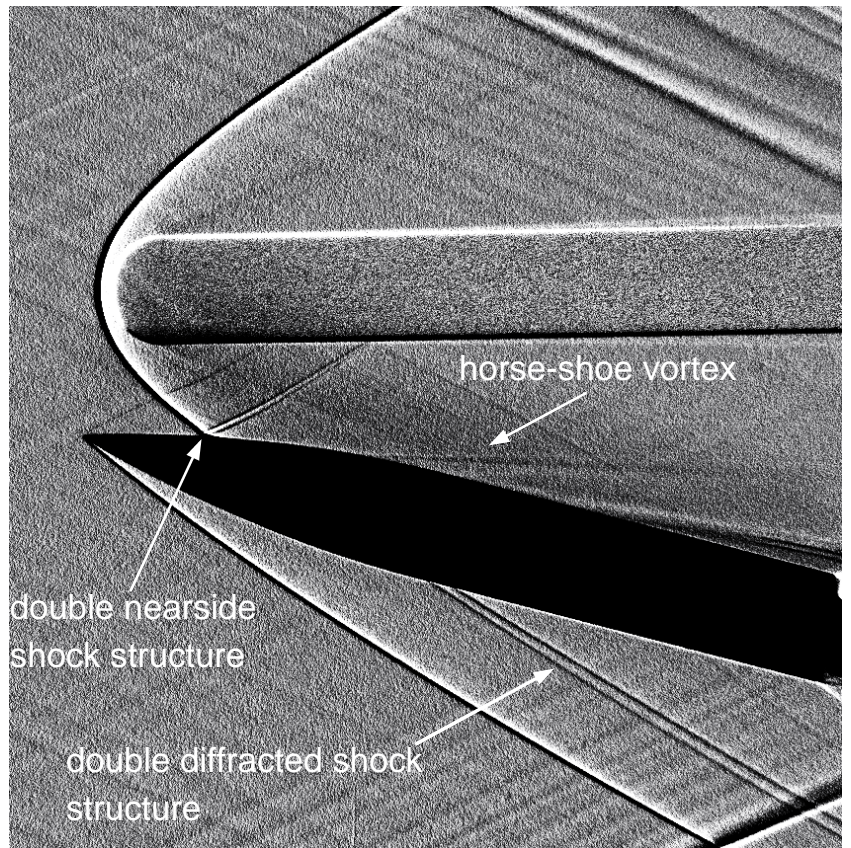

Fig. 28 Measured shadowgraph visualisation: blunt generator, $\Delta \mathrm{x} / \mathrm{D}=-0.53 \Delta \mathrm{z} / \mathrm{D}=2.94, \sigma_{\mathrm{R}}=15^{\circ} \sigma_{\mathrm{G}}=0^{\circ}$

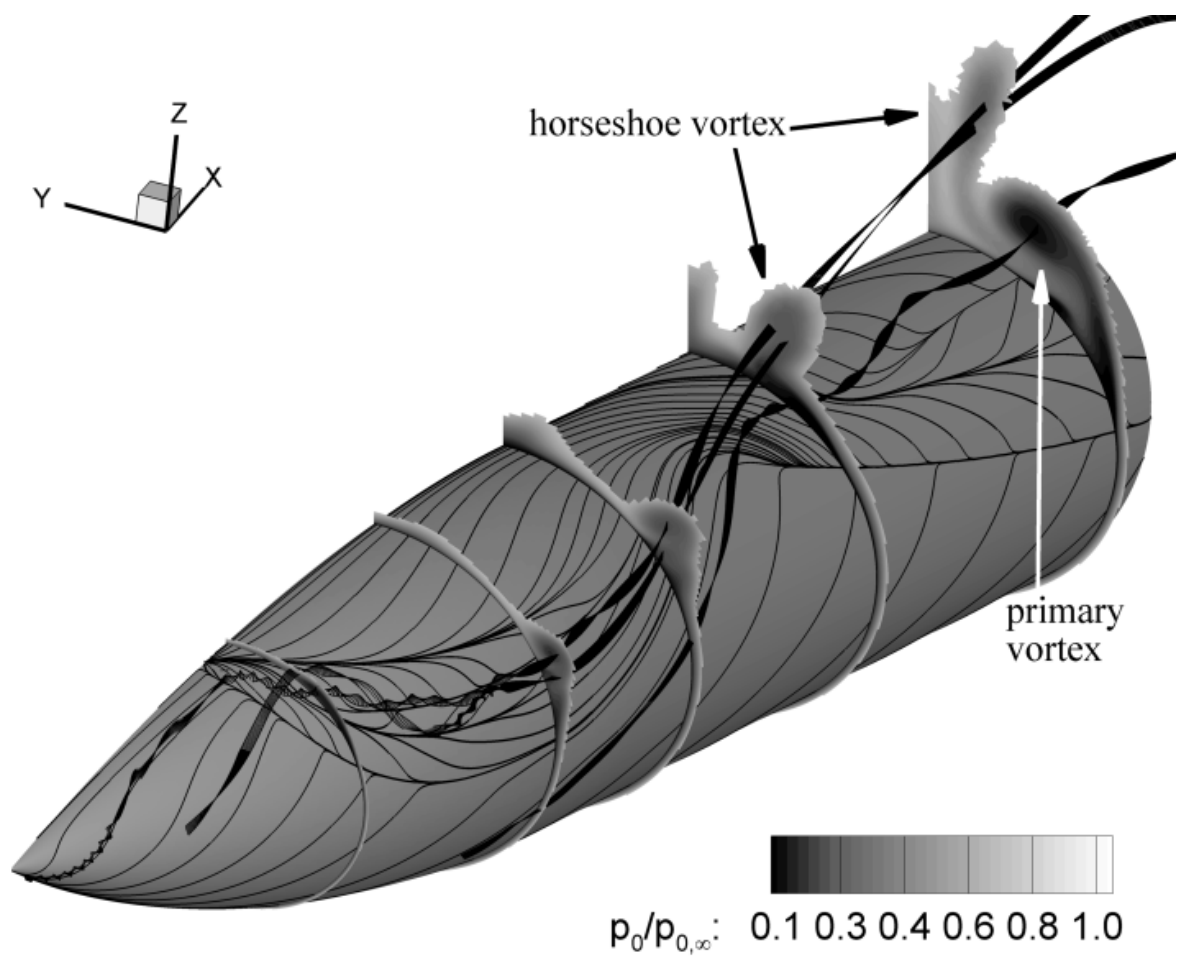

(a) Surface skin friction vector lines, contours of $p_{0} / p_{0, \infty}$ on crossflow planes and streamtrace ribbons 


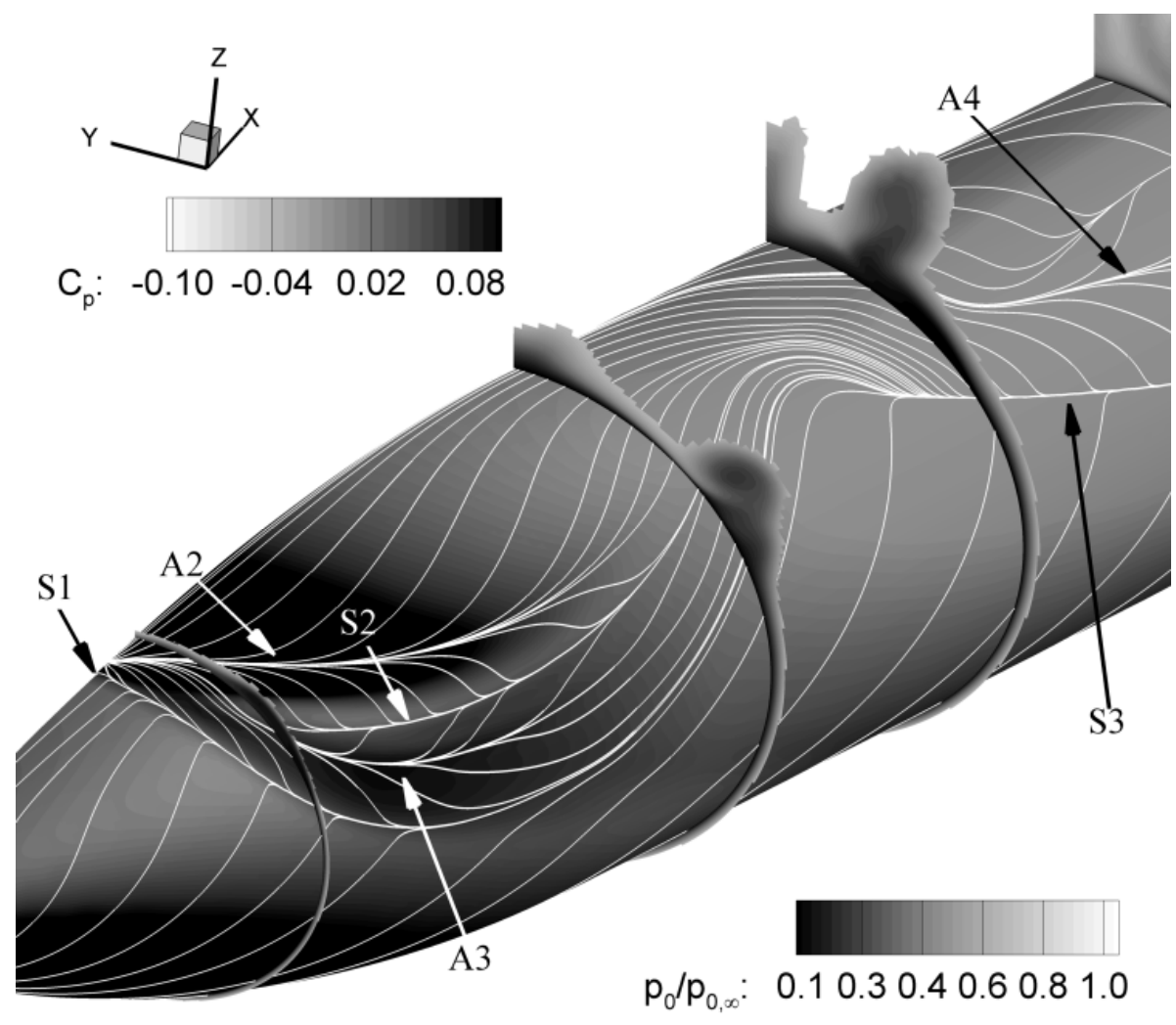

(b) Surface skin friction vector lines, crossflow slices of total pressure $\left(p_{0} / p_{0, \infty}\right)$ contours, surface pressure contours of static pressure coefficient $\left(\mathrm{C}_{\mathrm{p}}\right)$

Fig. 29 Predicted flowfield features highlighting the viscous flow structure: blunt generator, $\Delta x / D=-0.53$ $\Delta \mathrm{z} / \mathrm{D}=2.94, \sigma_{\mathrm{R}}=15^{\circ} \sigma_{\mathrm{G}}=0^{\circ}$

\subsubsection{Body vortex roll-up location}

The impinging shock causes a substantial re-organisation of the body crossflow characteristics due to the local separation region. This is because the shock footprint drives the flow in the opposite direction relative to the natural crossflow due to incidence and as a result there is a change in the crossflow separation location (Fig. $30 \mathrm{a}$ ). This roll-up location is much further aft than both the isolated configuration (Fig. $30 \mathrm{c}$ ) and the equivalent multibody configuration using the sharp generator where there is not a strong interaction (Fig. 30 b). Therefore, the presence of the horse-shoe vortex system and the characteristic differences in body vortex development has a notable effect on the receiver pressure distribution. The decrease in area over which the body vortices act reduces the vortex-lift component of the normal force compared with the sharp generator configuration (with a weak interaction) and the isolated configuration (with no interaction). Moreover, the primary vortex is smaller and closer to the surface (Fig. 29 a) in comparison to the isolated configuration. 


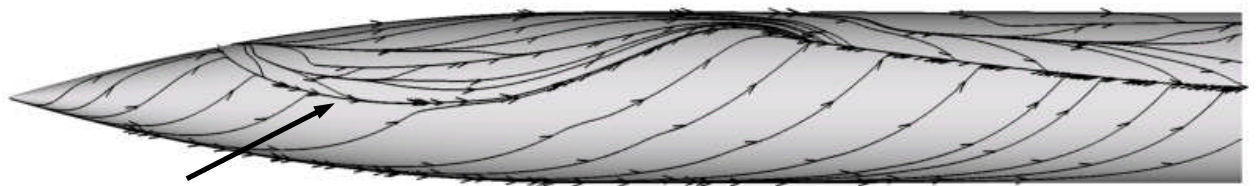

substantial flow re-organisation

(a) blunt generator: blunt generator, $\Delta \mathrm{x} / \mathrm{D}=-0.53 \Delta \mathrm{z} / \mathrm{D}=2.94, \sigma_{\mathrm{R}}=15^{\circ} \sigma_{\mathrm{G}}=0^{\circ}$
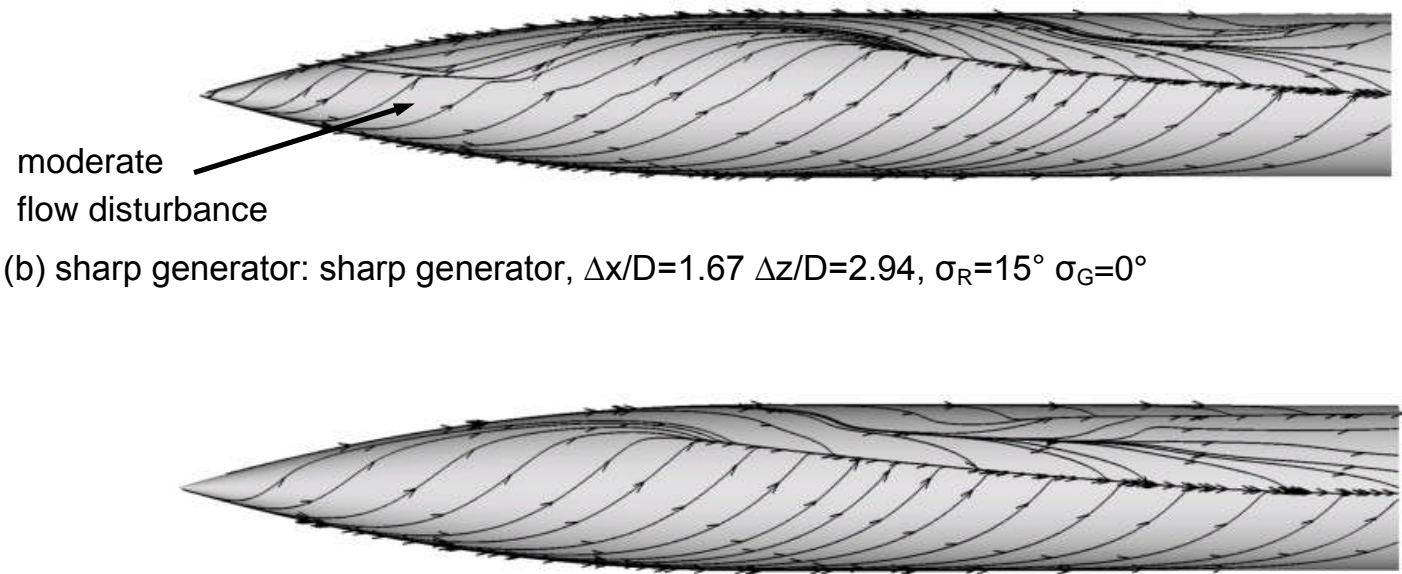

(c) isolated: $\sigma_{\mathrm{R}}=15^{\circ}$

Fig. 30 Predicted receiver surface skin friction vector lines for different configurations

\subsubsection{Effect on diffracted shockpath}

The differences discussed above due to the strong viscous interaction are important as it affects the surface pressure distribution and thus the body loads. However, there is another important observation for this configuration. The impinging shock diffracts to the farside further forward in comparison with the configuration using the sharp generator. The natural shock path for the equivalent configuration with the sharp generator is for the diffracted shock to exit through the receiver base (Fig. 10 a) and consequently there is no effect on the receiver farside. In the blunt generator configuration, however, the nearside separation region acts as an obstacle to the flow and a pre-compression shock forms forward of the separation region and the primary impinging shock. Both of these shockwaves then diffract to the farside where the dual shock structure on the receiver farside can be seen in Fig. 28 a. Finally, in the blunt generator configuration a farside region of positive differential pressure acts to offset the negative change in normal force induced by the nearside region of differential pressure.

\section{Conclusions}

The aerodynamics of high-speed interference between slender bodies have been investigated through a compliment of experimental and computational means. Few previous investigations have assessed the capability of computational fluid dynamics (CFD) to predict the complex flowfields involved in mutual interference at high-speeds. The predicted solutions in this paper have been successfully used to help interpret the measured results and to develop a deeper understanding of the underlying flow physics. This study has evaluated, in detail, he interference aerodynamics for slender bodies at incidence as well as the effect of a stronger disturbance field on the body of interest. Furthermore, analysis of the 
highly complex viscous interactions encountered when the receiver bodyis at high incidencehas been presented. The specific conclusions are discussed below in more detail.

In general, the CFD predictions are in good agreement with the measured results for all configurations. The CFD predicts the important flow features of the disturbance flowfield, the interference loads and the surface pressure distributions on the receiver body. A key aspect of this work has been the combined use of the experimental and computational data. The measurements provide a validation dataset for the CFD based on some of the key parameters. However, it is the additional field data from the computations that enables a fuller interpretation of the measurements and a better understanding of the associated flow physics.

The interference aerodynamics exhibit a variety of complex features which depend on the specific configuration arrangements. The axial impingement location of the generator bow shock has a profound effect on the magnitude and polarity of the interference loads.. In general, when the impingement location $\left(x^{\prime} / L\right)$ is near the receiver leading edge there is generally an attractive normal force interference $\left(\Delta \mathrm{C}_{Z}>0\right)$ and a negative pitching moment interference $\left(\Delta \mathrm{C}_{\mathrm{m}}<0\right)$. As the impingement location moves aftward, there is a change in polarity of both interference loads which leads to a repulsive normal force $\left(\Delta C_{Z}<0\right)$ when $x^{\prime} / L$ is towards the trailing edge.

Distinct interference characteristics are found when the receiver body is placed at $\sigma_{R}=15^{\circ}$. For these configurations, the diffracted shock does not reach the receiver farside which is a result of the shock obliqueness angle and geometric arrangement. Consequently, the effect of axial stagger is much more case specific and the induced changes in normal force and pitching moment are on-monotonic. It is observed, however, that the resulting interference loads are dependent on the main impingement point $\left(x^{\prime} / L\right)$ and whether this is fore or aft of the body vortex roll-up location. The largest observed interference effects are in the order of $\Delta \mathrm{C}_{\mathrm{z}}=-0.24\left(\sigma_{\text {eff }}=-1.8^{\circ}\right)$ and $\Delta \mathrm{C}_{\mathrm{m}}=1.1\left(\sigma_{\text {eff }}=-2^{\circ}\right)$.

Viscous shock interactions can have a significant impact on the leeside flow structure and consequently on the receiver body pressure distribution. A shock-induced horse-shoe vortex system is observed in a number of configurations as well as a delay in the roll-up of the primary body vortices. These effects alter the local normal force distribution along the body and thus change the interference loads.

Overall, the aerodynamic interference effects between two bodies in close proximity can be significant. The observed interference loads reported in this paper are expected to change the subsequent trajectories of the two slender bodies when the isolated loads are of the same order of magnitude. This is the case at low incidence (typically $\sigma_{R}=4^{\circ}$ or less) and is particularly important when the primary disturbance impinges near the trailing edge of the receiver body. In such a situation, relatively large pitch-up moments are induced and the likelihood of a collision is increased.

\section{Acknowledgements}

The authors would like to thank the UK MOD Defence Science and Technology Laboratory for both funding and providing technical support for this research. The first author was also partially funded through an Industrial CASE award from the UK Engineering and Physical Sciences Research Council. 


\section{References}

[1] Wilcox, F.: Separation Characteristics of Generic Stores from Lee Side of an Inclined Flat Plate at Mach 6. NASA TM 4652 (1995)

[2] Hung, C.: Computation of Three-Dimensional Shock Wave and Boundary-Layer Interactions. NASA TM 86780 (1985)

[3] Gapcynski, J., Carlson, H.: A Pressure-Distribution Investigation of the Aerodynamic Characteristics of a Body of Revolution in the Vicinity of a Reflection Plane at Mach Numbers of 1.41 and 2.01. NACA RM L54J29 (1955)

[4] Cenko, A., Waskiewicz, J.: Recent improvements in prediction techniques for supersonic weapon separation. J. Aircraft 20 (8), 659-666 (1983)

[5] Newman, G., Fulcher, K., Ray, R., Pinney, M.: On the aerodynamics/dynamics of Store Separation from a Hypersonic Aircraft. AIAA Paper 1992-2722 (1992)

[6] Chaplin, R.: Aerodynamic Interference between High-Speed Slender Bodies, PhD Thesis, Cranfield University UK (2009)

[7] Volkov, V., Derunov, E.: Interaction of a combination of bodies in supersonic flow. interference and diffraction of shock waves in flow over two bodies of revolution. J. Eng. Phys. Thermophys. 79 (4), 712-721 (2006)

[8] Malmuth, N., Shalaev, V.: Theoretical Modelling of Interaction of Multiple Slender Bodies in Supersonic Flows. AIAA Paper 2004-1127 (2004)

[9] Gapcynski, J.: The Aerodynamic Characteristics of a Body in the Flow Field Near the Tip of a Circular-Arc Wing of Rectangular Plan Form at a Mach Number of 2.01. NASA TM X$211(1960)$

[10] Waskiewicz, J., DeJongh, J., Cenko, A.: Application of panel methods to external stores at supersonic speeds. J. Aircraft 20 (2), 153-158 (1983)

[11] Fedorov, A., Malmuth, N., Soudakov, V.: Supersonic scattering of a wing-induced incident shock by a slender body of revolution. J. Fluid Mech. 585 305-322 (2007)

[12] Derunov, E., Zheltovodov, A., Maksimov, A.: Development of three-dimensional turbulent separation in the neighborhood of incident crossing shock waves. Th\&Ae. 15 (1), 29-54 (2008)

[13] Fedorov, A., Soudakov, V., Malmuth, N.: Theoretical modeling of two-body interaction in supersonic flow. AIAA J. 48 (2), 258-266 (2010)

[14] Brosh, A., Kussoy, M., Hung, C.: Experimental and numerical investigation of a shock wave impingement on a cylinder. AIAA J. 23 (6), 840-846 (1985)

[15] Morkovin, M., Migotsky, E., Bailey, H., Phinney, R.: Experiments on interaction of shock waves and cylindrical bodies at supersonic speeds. J. Aerosp. Sci. 19 (4), 237-247 (1952) 
[16] Hung, C.: Impingement of an Oblique Shock Wave on a Cylinder. AIAA Paper 19820025 (1982)

[17] Chaplin, R., MacManus, D., Birch, T.: Shock Waves 20 (2), 89-101 (2010)

DOI:10.1007/s00193-009-0241-7

[18] Taylor, J.: An Introduction to Error Analysis. University Science Books, California USA (1997)

[19] Cobalt LLC: Cobalt Users Manual (2007)

[20] Strang, W., Tomaro, R., Grismer, M.: The Defining Methods of Cobalt 60 : A Parallel, Implicit, Unstructured Euler/Navier-Stokes Solver. AIAA Paper 1999-0786 (1999)

[21] Menter, F.: Zonal Two Equation k-w Turbulence Models for Aerodynamic Flows. AIAA Paper 1993-2906 (1993)

[22] Pointwise: Gridgen (2006)

[23] Cummings, R., Morton, S., McDaniel, D.: Experiences in accurately predicting timedependent flows. Prog. Aerosp. Sci. 44 (4), 241-257 (2008)

[24] Roache, P.: Verification and Validation in Computational Science and Engineering. Hermosa Publishers, New Mexico USA (1998)

[25] Brosh, A., Kussoy, M.: An Experimental Investigation of the Impingement of a Planar Shock Wave on an Axisymmetric Body at Mach 3. NASA TM 84410 (1983) 